\title{
A Method for Determining the Uncertainty of Gap Conductance Deduced from Measured Fuel Centerline Temperatures
}

February 1977

Prepared for the

U.S. Nuclear Regulatory Commission 
NOTICE

This report was prepared as an account of work sponsored by the United States Government. Veither the United States nor the United States Nuclear Regulatory Commission, nor any of their employees, nor any of their contractors, subcontractors, or their employees, makes any warranty, express or implied, or assumes any legal liability or responsibility for the accuracy, completeness or usefulness of any information, apparatus, product or process disclosed, or represents that its use would not iniringe privately owned rights.

\author{
PACIFIC NORTHWEST LABORATORY \\ operated by \\ BATTELLE \\ for the \\ ENERGY RESEARCH AND DEVELOPMENT ADMINISTRATION \\ Under Contract EY-76-C-06-1830
}
Printed in the United States of America
Available from
National Technical Intormation Service
U.S. Department of Commerce
5285 Port Royal Road

Speringfieid, Virginia 22151

Price: Printed Copy $5 \_$__ Microtiche $\$ 3.00$

$\begin{array}{cc}\text {-Pages } & \text { NTIS } \\ \text { Seiling Price } \\ 007-025 & \$ 4.50 \\ 026-050 & \$ 5.00 \\ 051-075 & 55.50 \\ 076-100 & 56.00 \\ 101-125 & 56.50 \\ 126-150 & 57.00 \\ 151-175 & 57.75 \\ 176-200 & 58.50 \\ 201-225 & 58.75 \\ 226-250 & 59.00 \\ 251-275 & 510.00 \\ 276-300 & 510.25\end{array}$




\section{A METHOD FOR DETERMINING THE UNCERTAINTY OF GAP CONDUCTANCE DEDUCED FROM MEASURED FUEL CENTERLINE TEMPERATURES}

by

C. R. Hann, D. D. Lanning, R. K. Marsha17, A. R. 01sen and R. E. Williford

February 1977

BATTELLE

Pacific Northwest Laboratories

Richland, Washington 99352 


\section{SUMMARY}

This paper describes the method which was developed to determine the uncertainties of gap conductances deduced from measured fuel centerline temperatures of NRC-RSR/BPNL fuel rods irradiated in the Halden Boiling Water Reactor.

The $\int k(t) d t$ method is used to calculate the fuel surface temperature from the measured fuel centerline temperature and the fuel thermal conductivity. The gap conductance is calculated from the fuel surface temperature, the calculated cladding inside surface temperature, and the measured fuel assembly power. The uncertainties in the input parameters for calculating the gap conductance were established and the uncertainty in the gap conductance was calculated using the method of propagation of uncertainties with a first order Taylor series approximation to the nonlinear functions. An example of the calculational method is given. 


\section{CONTENTS}

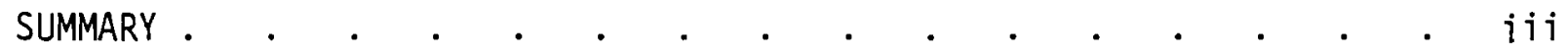

LIST OF FIGURES . . . . . . . . . . . . . . . . . v vii

LIST OF TABLES . . . . . . . . . . . . . . . . . . .

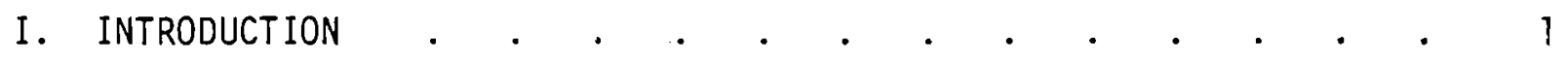

II. SUMMARY AND CONCLUSIONS . . . . . . . . . . . . . . . . 3

III. DESCRIPTION OF IRRADIATION TESTS .

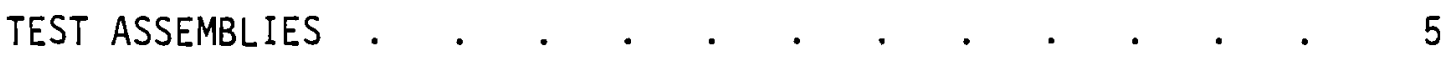

FUEL RODS . . .

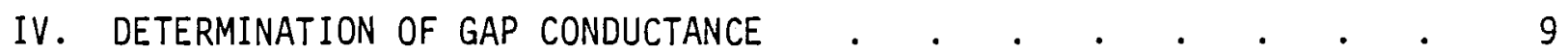

V. METHOD OF CALCULATING GAP CONDUCTANCE UNCERTAINTY . . . . . 13

V.A. CALCULATION OF UNCERTAINTY FOR THE

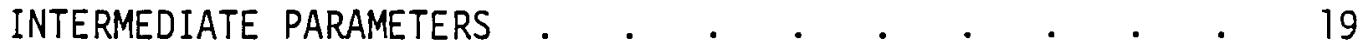

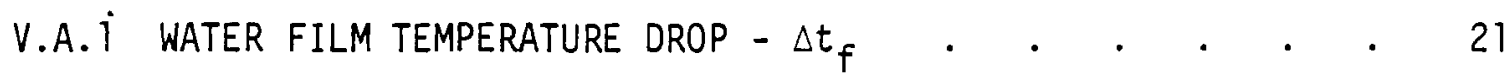

V.A.2 CLADDING TEMPERATURE DROP $-\Delta t_{c} \cdot \cdot \cdot \cdot \cdot \cdot \cdot \cdot \cdot \cdot \cdot 23$

V.A.3 CLADDING INSIDE SURFACE TEMPERATURE $-t_{1} \cdot \cdot \cdot \cdot \cdot \cdot 25$

V.A.4 FUEL SURFACE TEMPERATURE $-t_{s}$. . . . . . . . . 27

V.A.5 GAP TEMPERATURE DROP: $\Delta \mathrm{t}_{\mathrm{g}} \cdot \cdot \cdot \cdot \cdot \cdot \cdot \cdot \cdot \cdot \cdot \cdot \cdot \cdot \cdot 29$

V.B. CALCULATION OF UNCERTAINTY FOR GAP CONDUCTANCE . . . 31

VI. APPLICATION TO IFA-431 . . . . . . . . . . . . . . . . 37

REFERENCES . . . . . . . . . . . . . . . . . 43

APPENDIX A - Discussion of Assigned Uncertainty Values . . . . A-1

APPENDIX B - Examples of Relative Variance Calculations . . . B-1

APPENDIX C - Monte Carlo Sampling . . . . . . . . . $C-1$

APPENDIX D - Parametric Studies . . . . . . . . . D-1 


\section{LIST OF FIGURES}

1. Schematic of Instrumented Fuel Assembly--IFA-431 . . . . . . 7

2. Flow Chart for Determining Gap Conductance . . . . . . . . 11

3. Fuel Thermal Conductivity . . . . . . . . . . . . . 17

4. Curve Developed from A11 Data Except Lyons, et a1., Above $1600^{\circ} \mathrm{C}$ Plotted with A11 485 Data Points . . . . . . . . . 18

5. Gap Conductance Uncertainty Versus Linear Power for Rods 1, 5, and 6, IFA-431 . . . . . . . . . . . . . . . . . 38

6. Gap Conductance Uncertainty Versus Linear Power for Rod 2, IFA-431 . . . . . . . . . . . . . . . . . . . 39

7. Gap Conductance Uncertainty Versus Linear Power for Rod 3, IFA-431 . . . . . . . . . . . . . . . . . . . 40

8. Gap Conductance Uncertainty Versus Linear Power for Rod 4, IFA-431 . . . . . . . . . . . . . . . . . . . . . . . 41

9. Gap Conductance Versus Linear Power for Rods 1-6, IFA-431 . . . 42

\section{LIST OF TABLES}

1. HBWR Operating Data . . . . . . . . . . . . . . . 5

2. Design Parameters and Instrumentation for IFA-431 and IFA-432 . 6

3. Input Parameters and Uncertainties for IFA-431 . . . . . . 14

4. Water Film Temperature Drop Uncertainty: Example . . . . . 21

5. Cladding Temperature Drop Uncertainty: Example . . . . . . 23

6. Cladding Inside Surface Temperature Uncertainty: Example . . . 25

7. Fuel Surface Temperature Uncertainty: Example . . . . . . 28

8. Gap Temperature Drop Uncertainty: Example . . . . . . . . 29

9. Gap Conductance Uncertainty: Symmetric Example . . . . . . 32

10. Gap Conductance Uncertainty: Nonsymmetric Example . . . . . 35 


\section{INTRODUCTION}

The Battelle Pacific Northwest Laboratories are conducting irradiation tests in the Halden Boiling Water Reactor (HBWR) under the sponsorship of the Fuel Behavior Research Branch of the Nuclear Regulatory Commission. The objectives of the program are to 1) provide we11-characterized data for verifying computer codes used for reactor safety analysis and 2) develop improved analytical models for portions of these codes.

To assist in accomplishing these objectives, two test assemblies, each containing six instrumented fuel rods similar in design to BWR-type rods, were irradiated in the HBWR to obtain well-characterized data for fuel operating in the linear heat ranges of commercial nuclear power plants. These data are needed for verification of GAPCON-THERMAL ${ }^{(1)}$ and FRAP ${ }^{(2)}$ computer codes and will provide a series of benchmarks for indexing other thermal performance codes used for reactor safety analysis.

Parameters in the test include pellet-cladding gap size and eccentricity, fill gas composition, fuel density and stability, linear heat rating, and burnup. The two assemblies are essentially identical in design with one operating at a linear heat rating (LHR) of $10 \mathrm{~kW} / \mathrm{ft}(328 \mathrm{~W} / \mathrm{cm})($ IFA-431) and the other operating at $15 \mathrm{~kW} / \mathrm{ft}(492 \mathrm{~W} / \mathrm{cm})$ (IFA-432).

This report presents a method for estimating uncertainty on gap conconductances inferred from measured fuel centerline temperatures. The derivation of the method is presented in sufficient detail to permit the reader to substitute his own estimates of input uncertainties. Additional correction factors and associated uncertainties may also be incorporated with a minimum of difficulty. 


\section{SUMMARY AND CONCLUSIONS}

This report presents the method developed for estimating the reliability of gap conductances deduced from measured centerline fuel temperatures. In arriving at reliability estimates for experimental data, the ideal situation is to repeat al1 measurements of the input parameters required in the calculations enough times using enough observers and enough diverse instruments so that the reliability of the results can be assured by the use of statistics. Unfortunately, it is not practical to estimate all of the uncertainties of the input parameters by repetition. Since statistics could not be applied to the errors, the uncertainty intervals selected are based on past experience and engineering judgment. The uncertainty, which is the maximum deviation expected, is interpreted to be equivalent to $\pm 3 \sigma$ on a Gaussian distribution and hence includes $99.75 \%$ of the distribution.

The gap conductances for the fuel rods were deduced from measured centerline fuel temperatures using the $\int k(t) d t$ method $^{(3)}$ to calculate the fuel surface temperatures. The uncertainties in the gap conductances were calculated by utilizing the method of propagation of uncertainties with a first order Taylor series approximation to the nonlinear functions. Monte Carlo sampling was used to check the results.

Major conclusions of this study are:

1. Assuming normal distribution for all input variables, the distribution of deduced $\Delta T_{\text {gap }}$ is normal, but the distribution of gap conductance, $h_{\text {gap }}$, is skewed in general.

2. Feiller's method provides a reliable means of calculating nonsymmetric confidence intervals for $h_{\text {gap }}$.

3. The three dominant variables influencing the uncertainty in $\mathrm{h}_{\text {gap }}$ are centerline temperature, local linear heat rating and flux depression with fuel conductivity running a poor fourth. This occurred because of low uncertainty on 1 inear heat rating and fuel conductivity for this particular experiment. In general uncertainty on conductivity and heat rating would dominate the uncertainty in $h_{\text {gap }}$. 


\section{DESCRIPTION OF IRRADIATION TESTS}

Two test assemblies were irradiated in different channels in the Halden Boiling Water Reactor (HBWR) located in Norway. The reactor is a natural circulation reactor which currently operates at a power level of $12 \mathrm{MW}$. The reactor operating data are shown in Table 1.

\section{TABLE 1. HBWR Operating Data}

Power Leve 1

Reactor Pressure

Heavy Water Saturation Temperature

Plenum Inlet Temperature

Thermal Flux

Fast Flux $(>1 \mathrm{MeV})$

Average Fuel Power Density
$12 \mathrm{MW}$

500 psi (34 atm)

$464^{\circ} \mathrm{F}\left(240^{\circ} \mathrm{C}\right)$

$459^{\circ} \mathrm{F}\left(237^{\circ} \mathrm{C}\right)$

$23 \times 10^{13} \mathrm{n} / \mathrm{cm}^{2}-\mathrm{sec}$

$u 5 \times 10^{11} \mathrm{n} / \mathrm{cm}^{2}-\mathrm{sec} / \mathrm{W} / \mathrm{g}$

$14.8 \mathrm{~W} / \mathrm{g}$

\section{$\underline{\text { TEST ASSEMBLIES }}$}

The two assemblies, each containing a cluster of six instrumented fuel rods, are essentially identical in design with one operating at a linear heat rating (LHR) of $10 \mathrm{~kW} / \mathrm{ft}(328 \mathrm{~W} / \mathrm{cm})($ IFA-431) and the other operating at $15 \mathrm{~kW} / \mathrm{ft}(492 \mathrm{~W} / \mathrm{cm})$ (IFA-432). IFA-431 began operation in June of 1975 and was discharged after reaching a goal burnup of 4000 MWd/MTM in February of 1976. IFA-432 was charged into the reactor in December 1975 and wi11 run until 1ate 1977. The design parameters and instrumentation for the test assemblies are shown in Table 2.

Test assembly instrumentation includes six vanadium neutron detectors to monitor the neutron flux adjacent to the fuel rods and one cobalt neutron detector to monitor the neutron flux changes during power transients.

Test channel instrumentation includes inlet and outlet channel thermocouples, turbine flow meters, and a fuel rod failure detector. A schematic showing IFA-431 in its test channel is shown in Figure 1. IFA-432 is identical except for an ultrasonic thermometer in the upper end of Rod 2. 
TABLE 2. Design Parameters and Instrumentation for IFA-431 and IFA-432

\begin{tabular}{|c|c|c|c|c|c|c|c|c|c|c|c|c|}
\hline \multirow{3}{*}{$\begin{array}{l}\text { Rod } \\
\text { No. }\end{array}$} & \multirow{2}{*}{\multicolumn{2}{|c|}{ Pellet OD }} & \multirow{2}{*}{\multicolumn{2}{|c|}{$\begin{array}{c}\text { Cold } \\
\text { Diametral Gap }\end{array}$}} & \multirow{3}{*}{\multicolumn{2}{|c|}{$\begin{array}{l}\text { Fue } 1 \\
\text { Density } \\
\% \text { TD } \\
\end{array}$}} & \multirow{3}{*}{$\begin{array}{l}\text { Fuel } \\
\text { Type }\end{array}$} & \multirow{3}{*}{$\begin{array}{l}\text { Burnup } \\
\text { Mild/MTM }\end{array}$} & \multicolumn{4}{|c|}{ Instrumentation } \\
\hline & & & & & & & & & \multicolumn{2}{|c|}{ Temperature } & \multirow{3}{*}{$\frac{\text { Pressure }}{P_{T}(b)}$} & \multirow{2}{*}{$\begin{array}{l}\text { Cladding } \\
\text { Length }\end{array}$} \\
\hline & in. & $\mathrm{mm}$ & in. & $\mathrm{mm}$ & & & & & Lipper & Lower & & \\
\hline 1 & 0.4205 & 10.581 & 0.009 & 0.229 & $\mathrm{He}$ & 95 & Stable & 4,000 & $T C^{(a)}$ & TC & & $E S^{(c)}$ \\
\hline 2 & 0.4145 & 10.528 & 0.015 & 0.381 & $\mathrm{He}$ & 95 & Stable & 4,000 & $T C$ & TC & -- & ES \\
\hline 3 & 0.4275 & 10.858 & 0.002 & 0.051 & $\mathrm{He}$ & 95 & Stable & 4,000 & TC & TC & -- & ES \\
\hline 4 & 0.4205 & 10.681 & 0.009 & 0.229 & $\mathrm{Xe}$ & 95 & Stable & 4,000 & TC & TC & -- & ES \\
\hline 5 & 0.4205 & 10.681 & 0.009 & 0.229 & $\mathrm{He}$ & 92 & Stable & 4,000 & TC & TC & PT & ES \\
\hline 6 & 0.4205 & 10.681 & 0.009 & 0.229 & $\mathrm{He}$ & 92 & Unstable & 4,000 & TC & TC & PT & ES \\
\hline
\end{tabular}

IFA-432 - Peak Power - $15 \mathrm{~kW} / \mathrm{ft}(492 \mathrm{w} / \mathrm{cm})$

$\begin{array}{lllllll}2 & 0.4145 & 10.528 & 0.015 & 0.381 & \text { He. }\end{array}$

3. $0.4265 \quad 10.858 \quad 0.003 \quad 0.076$ He 95

$\begin{array}{llllllll}4 & 0.4205 & 10.681 & 0.009 & 0.229 & \text { Xe } & 95\end{array}$

$\begin{array}{lllllll}5 & 0.4205 & 10.681 & 0.009 & 0.229 & \text { He } & 92\end{array}$

$\begin{array}{lllllll}6 & 0.4205 & 10.681 & 0.009 & 0.229 & \text { He } & 92\end{array}$

$\begin{array}{lllllll}7 & 0.4145 & 10.528 & 0.015 & 0.381 & \text { He } & 95\end{array}$

$\begin{array}{lllllll}8 & 0.4205 & 10.587 & 0.009 & 0.229 & \text { He } & 95\end{array}$

$\begin{array}{lllllll}9 & 0.4225 & 10.732 & 0.007 & 0.179 & \mathrm{He}\end{array}$

95

$\begin{array}{lclccc}\text { Stable } & 20,000 & \text { TC } & \text { TC } & \text { PT } & \text { ES } \\ \text { Stable } & 4,000^{(d)} & \text { UT }(e) & \text { TC } & -- & \text { ES } \\ \text { Stable } & 4,000^{(d)} & \text { TC } & \text { TC } & -- & \text { ES } \\ \text { Stable } & 4,000^{(d)} & \text { TC } & \text { TC } & -- & \text { ES } \\ \text { Stable } & 20,000 & \text { TC } & \text { TC } & \text { PT } & \text { ES } \\ \text { Unstable } & 20,000 & \text { TC } & \text { TC } & \text { PT } & \text { ES } \\ \text { Stable } & 16,000 & -- & -- & -- & -- \\ \text { Stable } & 16,000 & -- & -- & -- & -- \\ \text { Stable } & 16,000 & -- & -- & -- & --\end{array}$

a. $T C=$ Thermocouple

b. $P T=$ Pressure Transducer

c. $E S=$ Elongation Sensor

d. Removable rod's replaced by rods 7,8 , and 9 .

e. $U T=$ UItrasonic Thermometer 


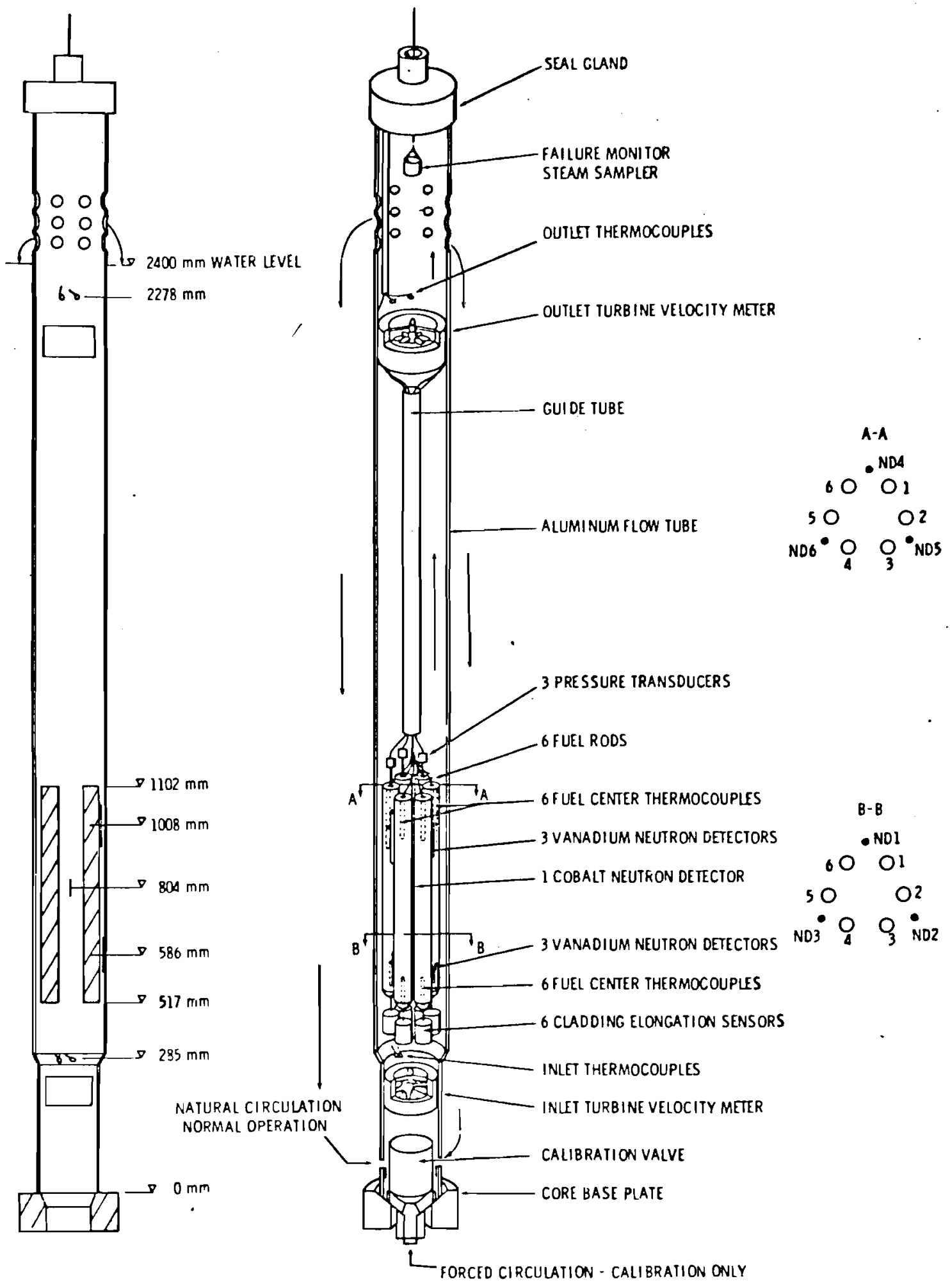

FIGURE 1. Schematic of Instrumented Fuel Assembly--IFA-431 
Data from the instrumentation except for the pressure monitors is automatically taken every 15 minutes by a data logging system and stored on magnetic tape. The pressure data are manually taken.

\section{FUEL RODS}

Each fuel rod is instrumented with two temperature sensors to measure the central fuel temperature and with one cladding elongation sensor to detect the time and extent of pellet-cladding interaction. All of the temperature sensors are thermocouples except for one in IFA-432, which is an ultrasonic thermometer (UT). Three rods in each assembly are each equipped with a pressure transducer to monitor the internal rod pressure. All rods contain $\mathrm{U}_{2}$ pellets enriched to $10 \mathrm{wt} \%{ }^{235} \mathrm{U}$. The pellets have flat ends and are $12.7 \mathrm{~mm}(0.5 \mathrm{in.})$ long.

Three of the test fuel rods (Rods 1, 5, and 6) were designed to measure the effect of fuel densification and stability on gap conductance. These rods had $0.051 \mathrm{~mm}(0.009 \mathrm{in.})$ cold gaps and were back-filled with helium at one atmosphere pressure. Rod 1 was the reference rod and contained 95\% theoretical density (TD) stable fuel. Rods 5 and 6 each contained 92\% TD fuel; however, the fuel in Rod 5 was stable while fuel in Rod 6 was unstable and thus susceptible to densification.

Rod 2 was designed to simulate instantaneous densification. Its design is identical to Rod 1 except for a gap of $0.381 \mathrm{~mm}$ (0.015 in.).

Rod 3 was designed primarily as an independent check of rod linear heat ratings. This rod is identical to Rod 1 except for a gap of $0.051 \mathrm{~mm}$ (0.002 in.) in IFA-431 and $0.076 \mathrm{~mm}$ (0.003 in.) in IFA-432. This gap width was chosen to provide good contact at power and yet minimize the potential for cladding failure induced by mechanical interaction. With the temperature gradient across the gap minimized and knowing the measured centerline fuel temperature and calculating the cladding temperature, the rod LHR in the assembly can be checked.

Rod 4 was designed to gain an insight into the anomalous thermal behavior of xenon-filled rods and to study the effects of pellet-cladding gap eccentricity. 


\section{DETERMINATION OF GAP CONDUCTANCE}

The pellet-cladding gap conductance in a fuel rod is defined by the following equation:

$$
h_{g}=\frac{\pi\left(d_{i}+d_{p}\right)}{2}\left(t_{s}-t_{j}\right)
$$

where: $h_{g}=$ pellet-cladding gap conductance

$q=1$ inear heat rating

$t_{s}=$ fuel surface temperature

$t_{j}=$ cladding inside surface temperature

$d_{j}=$ cladding inside diameter

$d_{p}=$ pellet diameter.

It is worthwhile to note that none of the variables in equation (1) have ever been measured directly in an operating fuel rod in this or any other irradiation test. The average gap diameter $\left(d_{i}+d_{p}\right) / 2$, is quite close to $i$ ts average for the as-fabricated dimensions; and the latter number is used. The cladding inner temperature $t_{i}$, can be calculated with little error from the following equations:

$$
t_{j}=t_{w}+\Delta t_{f}+\Delta t_{c}
$$

where: $\quad t_{j}=$ cladding inside surface temperature

$t_{w}=$ coolant temperature adjacent to point of measurement

$\Delta t_{f}=$ water film temperature drop

$\Delta t_{c}=c l$ adding temperature drop.

The water film temperature $\operatorname{drop}\left(\Delta t_{f}\right)$ and cladding temperature drop $\left(\Delta t_{c}\right)$ are expressed as:

$$
\begin{aligned}
\Delta t_{f} & =\frac{q}{\pi d_{0} h_{f}} \text { and } \\
\Delta t_{c} & =\frac{q \ln \left(d_{0} / d_{j}\right)}{2 b}
\end{aligned}
$$




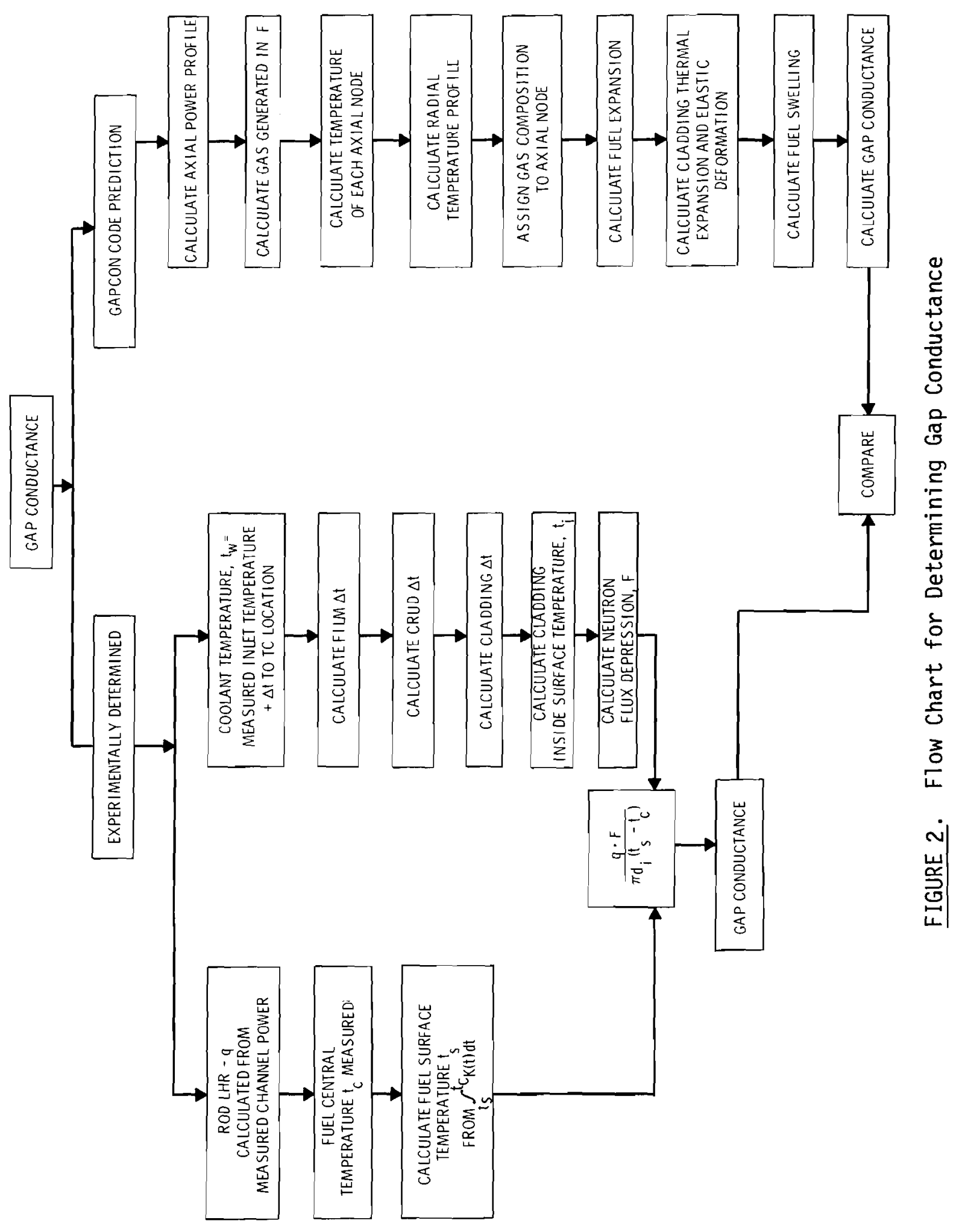


Note that the linear heat rating is the most influential parameter in calculating the gap conductance and uncertainty because it enters into the calculations three times. It appears in the calculation of the fuel surface temperature, the cladding ID temperature, and the gap conductance. The linear heat rating for each fuel rod is calculated from the measured fuel assembly power. 


\section{METHOD OF CALCULATING GAP CONDUCTANCE UNCERTAINTY}

After the gap conductances are deduced from the test data, their estimated uncertainties are calculated. This section presents the calculational methods employed and an example of their application.

A distinction between variability, due to measurement and other causes, and bias is necessary. For example, the linear heat rating value may not coincide with the true, but unknown, linear heat rating due to two different reasons. First, the process of determining the linear heat rating involves making measurements which are subject to error. If the process were repeated a number of times under the same conditions, a distribution of values would be obtained centered at some average value. This average value does not necessarily coincide with the actual true value. This difference is called the bias. The variability around the average value is the uncertainty as used in the following analysis. Hence, the uncertainty determined for the gap conductance is concerned with the variability described above and does not take into account any possible bias.

Appendix A traces the methods by which the major input variables for $h_{\text {gap }}$ calculations for IFA-431-432 were deduced. It also traces the assumptions involved and the assigrments of uncertainty. The final assignments of uncertainty are listed in Table 3 .

The uncertainty analysis is based on a few stringent assumptions. These are necessary in order to make any progress at all due to the lack of actual data concerning the variability of the input variables, e.g., diameters, temperatures and linear heat rating. The assumptions are:

1. For each input variable, not calculated by equations 1 to 5 , the error distribution is Gaussian about a center value $\mu$. Moreover, the uncertainty is adequately described by the relative variance $c^{2}=\sigma^{2} / \mu^{2}$. Note this implies that the variability increases with increases in the center value, i.e., the percent uncertainty is constant. 
TABLE 3. Input Parameters and Uncertainties for IFA-431

Fuel Rods

Uncertainty $\pm \%$

Linear heat rating

Rods 1-6 at lower thermocouple location and

Rods $1,2,3$ and 6 at upper thermocouple location

5.6

Rods 4 and 5 at upper thermocouple location

6.3

Fuel thermal conductivity $(a)$

Centerline temperature

\section{Thermocouple}

$1000^{\circ} \mathrm{F}\left(538^{\circ} \mathrm{C}\right)-4000^{\circ} \mathrm{F}\left(2204^{\circ} \mathrm{C}\right)$

$4000^{\circ} \mathrm{F}\left(2204^{\circ} \mathrm{C}\right)-4500^{\circ} \mathrm{F}\left(2482^{\circ} \mathrm{C}\right)$

3.0

4.0

Cladding thermal conductivity

5.0

Dimensions, density, enrichment

0.2

Neutronic

Thermal neutron flux depression in fuel

5.0

Thermal-Hydraulic

Water film heat transfer coefficient

Bulk water temperature

15.0

1.0

(a) See figures 3 and 4 and the discussion in Appendix A. 
2. There is zero covariance between any two of the input variables. In particular, this implies there is no correlation between the measurement errors on the input variables.

3. For the nonlinear functions of the input variables used in equations 1 to 5 , the propagation of errors by first order Taylor series provides an adequate approximation.

4. The distributions of the derived variables, e.g., $h_{g}$, are sufficiently symmetric that assumption 1 above holds for the derived variables.

Assumption 4 deserves additional explanation. The explanation will be given for fuel surface temperatures. Suppose that it were possible to conduct a large number of experiments exactly $1 \mathrm{ike}$ the IFA-431 test. Furthermore, assume that the necessary measurements needed to calculate the fuel surface temperatures are taken at the same point of time, i.e., same burnup, power, etc., for each of the experiments. The temperatures calculated under these conditions will form a distribution of values. Assumption 4 states that this distribution is approximately symmetric about the true value for the conditions of the experiment. All of the assumptions are concerned with this hypothetical experimental framework.

An elementary discussion of the Taylor's series approximation is given in Reference 4. The error propagation for the gap conductance follows the framework described by Jaech. (4) In the case of the fuel surface temperature, which is defined implicitly in an integral equation, a little ingenuity enables the variance of $t_{s}$ to be obtained without an explicit solution. The main idea is to recognize that the variances of the right and left hand sides must be equal. The details are given later.

The notation is in terms of relative variances. Let $w$ and $v$ be variables with standard deviations $\sigma_{w}$ and $\sigma_{v}$, means $\mu_{w}$ and $\mu_{v}$, and covariance $\sigma_{w v}$, respectively. The relative variance of $v$ is $C_{v}^{2}=\sigma_{v} 2 / \mu_{v}^{2}$ and the relative covariance of $w$ and $v$ is $C_{W V}=\sigma_{w V} / \mu_{W} \mu_{V}$. It is convenient at times to use $C_{W W}$ in place of $C_{W}{ }^{2}$ and $\sigma_{W W}$ in place of $\sigma_{W}{ }^{2}$. The square root of the relative variance $C_{W}$ is also called the coefficient of variation, i.e., $C_{W}=\sigma_{W} / \mu_{W}$. 
For the present assume that the information available consists of the necessary means, standard deviations and covariances. Where possible the equations will be in terms of the relative variances and covariances.

Engineering 1 imits, or uncertainties, require additional interpretation and/or assumptions before they are used. For example, what does the statement "the uncertainty of the cladding inside diameter is $\pm 0.03 \% "$ mean? One interpretation is that the error distribution is Gaussian and its standard deviation is $0.0003 d_{i} / 3$ where $d_{i}$ is the true value. The division by 3 corresponds to the assumption that the uncertainty gives the maximum deviation expected. Moreover, this maximum deviation is interpreted to be equivalent to $\pm 3 \sigma$ on the Gaussian distribution and, hence, includes $99.75 \%$ of the distribution. This is the interpretation assumed for the uncertainties quoted in the gap conductance problem.

The six output values of interest are $\Delta t_{f}, \Delta t_{c}, t_{i}, t_{s}, \Delta t_{g}$ and $h_{g}$, of which the first five are intermediate variables. The derivation of relative variances for the intermediate variables will now be performed. After each derivation a set of numerical values corresponding to a standard problem (Rod 1 at $3.0 \times 10^{4} \mathrm{~W} / \mathrm{m}$ ) will be given. 


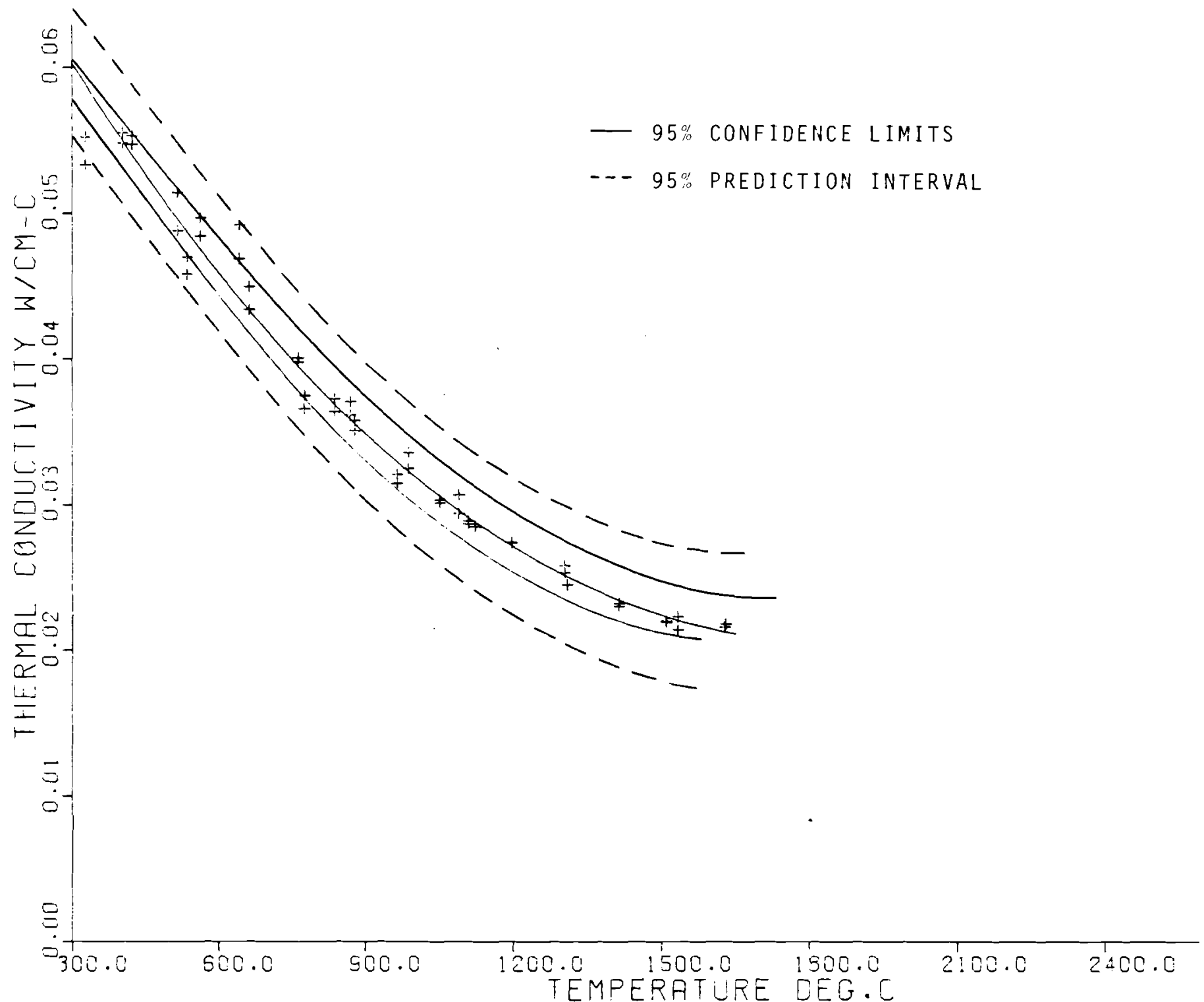

FIGURE 3. Fuel Thermal Conductivity (Ref. 5) 


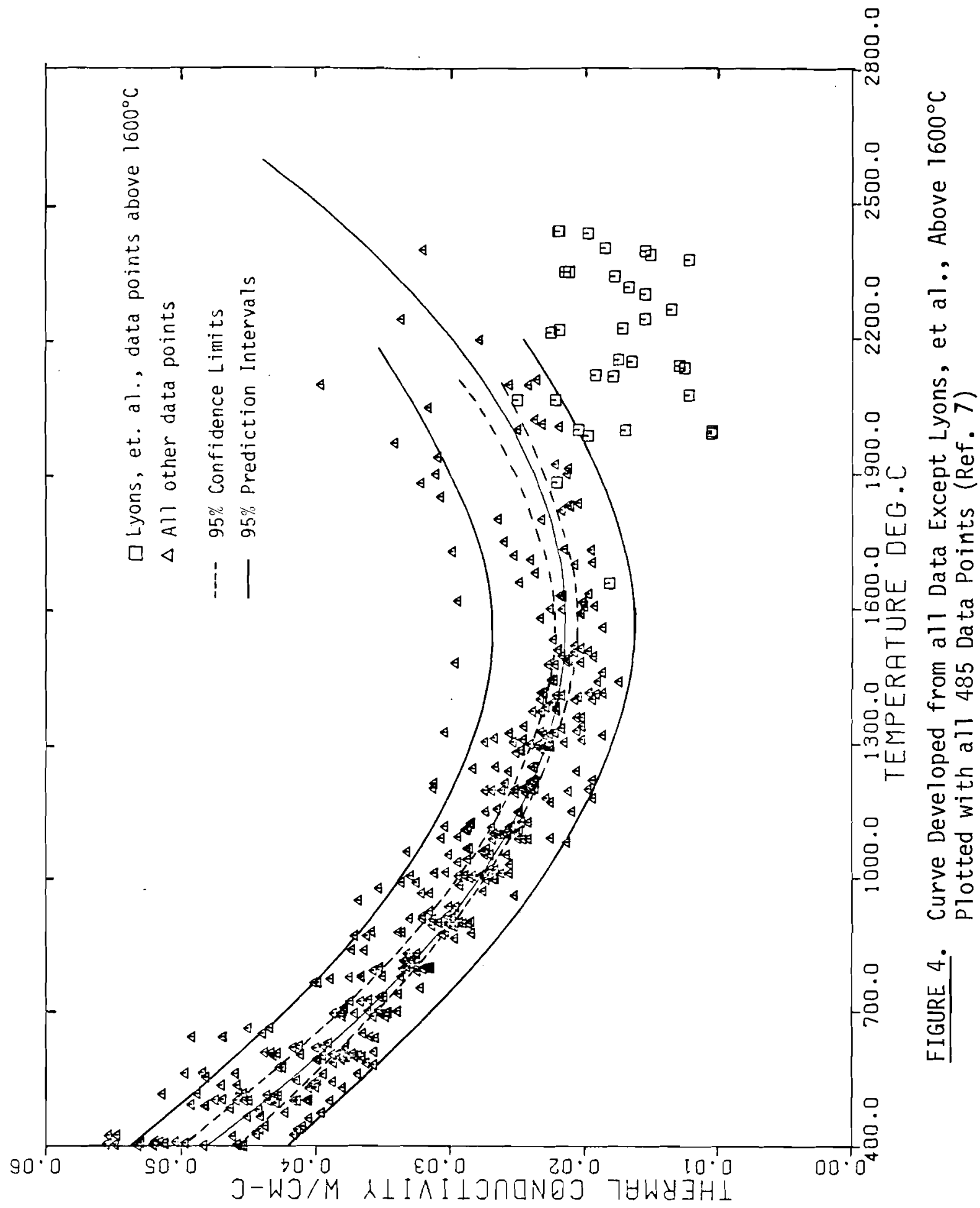


The five intermediate parameters in the gap conductance calculations are:

- water film temperature drop $\left(\Delta t_{f}\right)$,

- cladding temperature $\operatorname{drop}\left(\Delta \mathrm{t}_{\mathrm{c}}\right)$,

- inside cladding temperature $\left(t_{j}\right)$,

- fuel surface temperature $\left(t_{s}\right)$,

- gap temperature $\operatorname{drop}\left(\Delta \mathrm{t}_{\mathrm{g}}\right)$.

This section presents the error propagation equations for these intermediate parameters. A typical calculation is given in each case. 


\section{V.A.1. WATER FILM TEMPERATURE DROP $-\Delta t_{f}$}

The water film temperature drop is computed from

$$
\Delta t_{f}=\frac{q}{\pi d_{0} h_{f}}
$$

The relative covariance obtained from the error propagation is given by

$$
c_{\Delta t_{f}}^{2}=c_{q}^{2}+c_{d_{0}}^{2}+c_{h_{f}}^{2}
$$

where $c_{q}^{2}, c_{d_{0}}^{2}$ and $c_{h_{f}}^{2}$ are the relative variances of the linear heat rating, outside diameter of the cladding and the water film heat transfer coefficient. These quantities can be calculated from their assumed uncertainties by using $c_{x}^{2}=\left(V_{q} / 300\right)^{2}$. The uncertainty for the water film temperature drop is obtained from the reverse calculation. An example is given in Table 4.

\begin{tabular}{|c|c|c|c|c|}
\hline \multirow[b]{2}{*}{ Parameter } & \multirow[b]{2}{*}{ Value } & \multirow[b]{2}{*}{$\begin{array}{c}\text { Uncertainty, } \\
\%\end{array}$} & \multicolumn{2}{|c|}{ Contribution to $c_{\Delta t_{\bar{f}}}^{2}$} \\
\hline & & & Value & $\%$ of Total \\
\hline$q(W / m)$ & $3.0+04$ & 5.6 & $3.48-04$ & 12.2 \\
\hline$d_{0}(m)$ & $1.278-02$ & 0.2 & $4.44-07$ & $<0.01$ \\
\hline$h_{f}$ & 5.892 & 15.0 & $2.50-03$ & 87.8 \\
\hline$\Delta t_{f}$ & 12.680 & 16.0 & $2.85-03$ & 100.0 \\
\hline
\end{tabular}

TABLE 4. Water Film Temperature Drop Uncertainty: Example 


\section{V.A.2. CLADDING TEMPERATURE DROP $-\Delta t_{c}$}

The cladding temperature drop is computed from

$$
\Delta t_{c^{\prime}}=\frac{q \ln \left(d_{0} / d_{i}\right)}{2 \pi b}
$$

Its relative variance is obtained from

$$
c_{\Delta t_{c}}^{2}=c_{q}^{2}+c_{b}^{2}+\frac{c_{d_{0}}^{2}+c_{d_{i}}^{2}}{\left[\ln \left(d_{0} / d_{i}\right)\right]^{2}}
$$

where $c_{q}^{2}, c_{b}^{2}, c_{d_{0}}^{2}$ and $c_{d_{i}}^{2}$ are the relative variances of the linear heat rating, cladding thermal conductivity, cladding outside diameter and the cladding inside diameter respectively. Table 5 presents an example calculation.

\begin{tabular}{|c|c|c|c|c|}
\hline \multirow[b]{2}{*}{ Parameter } & \multirow[b]{2}{*}{ Value } & \multirow{2}{*}{$\begin{array}{c}\text { Uncertainty, } \\
\%\end{array}$} & \multicolumn{2}{|c|}{ Contribution to $c_{\Delta t}^{2}$} \\
\hline & & & Value & $\%$ of Total \\
\hline$q$ & $3.0+04$ & 5.6 & $3.48-04$ & 52.6 \\
\hline$b(W / m-C)$ & $1.535-03$ & 5.0 & $2.78-04$ & 42.0 \\
\hline$d_{0}(m)$ & $1.278-02$ & 0.2 & $1.76-05$ & 2.7 \\
\hline$d_{j}(m)$ & $1.090-02$ & 0.2 & $1.76-05$ & 2.7 \\
\hline$\overline{\Delta t}{ }_{c}$ & 49.4 & $\overline{7.7}$ & $\overline{6.62-04}$ & $\overline{100.0}$ \\
\hline
\end{tabular}

TABLE 5. Cladding Temperature Drop Uncertainty: Example 


\section{V.A.3. CLADDING INSIDE SURFACE TEMPERATURE $-\mathrm{t}_{i}$}

The cladding inside surface temperature is computed from

$$
t_{i}=t_{w}+\Delta t_{f}+\Delta t_{c}
$$

and its relative variance is

$$
c_{t_{i}}^{2}=\left(\frac{t_{w}}{t_{i}}\right)^{2} c_{t_{w}}^{2}+\left(\frac{\Delta t_{f}}{t_{i}}\right)^{2} c_{\Delta t_{f}}^{2}+\left(\frac{\Delta t_{c}}{t_{i}}\right)^{2} c_{\Delta t_{c}}^{2}+2 \frac{\Delta t_{f} \Delta t_{c}}{t_{i}^{2}} c_{\Delta t_{f}, \Delta t_{c}}
$$

where $c_{t_{w}}^{2}, c_{\Delta t_{f}}^{2}$, and $c_{\Delta t_{c}}^{2}$ are the relative variances of the bulk water temperature, water film temperature drop and the cladding temperature drop, respectively, and $c_{\Delta t_{f}}, \Delta t_{c}$ is the relative covariance between $\Delta t_{f}$ and $\Delta t_{c}$ obtained from

$$
c_{\Delta t_{f}, \Delta t_{c}}=c_{q}^{2}-\frac{c_{d_{0}}^{2}}{\left[\ln \left(d_{0} / d_{j}\right)\right]}
$$

In this case there is a relative covariance between $\Delta t_{f}$ and $\Delta t_{c}$ since they

\begin{tabular}{|c|c|c|c|c|}
\hline \multirow[b]{2}{*}{ Parameter } & \multirow[b]{2}{*}{ Value } & \multirow[b]{2}{*}{$\begin{array}{c}\text { Uncerta inty, } \\
\%\end{array}$} & \multicolumn{2}{|c|}{ Contribution to $c_{t}^{2}$} \\
\hline & & & Value & $\%$ of Total \\
\hline$t_{w}$ & 238. & 1.0 & $6.99-06$ & 20.1 \\
\hline$\Delta \mathrm{t}_{f}$ & 12.68 & 16.0 & $5.09-06$ & 14.6 \\
\hline$\Delta t_{c}$ & 49.4 & 7.7 & $1.79-05$ & 51.5 \\
\hline$\Delta t_{f}, \Delta t_{c}$ & & & $4.81-06$ & 13.8 \\
\hline$t_{i}$ & 300.00 & 1.8 & $3.48-05$ & 100.0 \\
\hline
\end{tabular}
are both functions of $q$ and $d_{0}$. Table 6 gives an example.

TABLE 6. Cladding Inside Surface Temperature Uncertainty: Example 


\section{V.A.4. FUEL SURFACE TEMPERATURE $-t_{s}$}

The fuel surface temperature is defined implicitly by

$$
\int_{t_{S}}^{t} c(t) d t=\frac{q F}{4 \pi}
$$

where $K(t)$ is a third degree polynomial with the coefficients estimated by linear regression techniques. Although it is theoretically possible to solve for $t_{s}$ explicitly, the solution is complicated. The uncertainty of $t_{s}$ may be approximated without an explicit solution. The error propagation requires partial derivatives of the form $\frac{\partial t_{s}}{\partial x}$ where $x$ represents any of the remaining variables. These derivatives can ${ }^{\partial X}$ be obtained by implicit differentiation. Otherwise the procedure is the same. The relative covariance of the fuel surface temperature is given by

$c_{t_{s}}^{2}=\left[\frac{t_{c} k\left(t_{c}\right)}{t_{s} k\left(t_{s}\right)}\right]^{2} c_{t_{c}}^{2}+\left[\frac{q F}{4 \pi t_{s} k\left(t_{s}\right)}\right]^{2}\left(c_{q}^{2}+c_{F}^{2}\right)+\frac{\left[x\left(t_{c}\right)-x\left(t_{s}\right)\right]^{\prime} \Sigma\left[x\left(t_{c}\right)-x\left(t_{s}\right)\right]}{\left(t_{s} k\left(t_{s}\right)\right)^{2}}$

where

$$
\begin{aligned}
K(t)= & a+b t+c t^{2}+d t^{3} \\
x(t)^{-}= & \left(t, \frac{1}{2} t^{2}, \frac{1}{3} t^{3}, \frac{1}{4} t^{4}\right), \text { and } \\
\Sigma= & \text { covariance matrix for regression } \\
& \text { coefficients of } K(t) .
\end{aligned}
$$

An example is given in Table 7. The value used for $K(t)$ and $\Sigma$ are

$$
\begin{aligned}
K(t)=0.0784- & (6.628-05) t+(2.083-08) t^{2}-(1.015-12) t^{3} \\
& \text { (Curve-fit from Figure 3) }
\end{aligned}
$$




$$
\Sigma=10^{-6}\left[\begin{array}{ccrr}
1.7639 & -78583-03 & 9.3653-06 & -3.2519-09 \\
-7.8583-03 & 4.1342-05 & -5.3056-08 & 1.9209-11 \\
9.3653-06 & -5.3056-08 & 7.1311-11 & -2.6624-14 \\
-3.2519-09 & 1.9209-11 & -2.6624-14 & 1.0170-17
\end{array}\right]
$$

TABLE 7. Fuel Surfaee Temperature Uncertainty: Example

\begin{tabular}{|c|c|c|c|c|}
\hline \multirow[b]{2}{*}{ Parameter } & \multirow[b]{2}{*}{ Value } & \multirow{2}{*}{$\begin{array}{c}\text { Uncertainty, } \\
\%\end{array}$} & \multicolumn{2}{|c|}{ Contribution to $c_{t}^{2}$} \\
\hline & & & Value & $\%$ of Total \\
\hline$t_{C}$ & 1040.0 & 3.0 & $1.53-04$ & 26.8 \\
\hline$q$ & $3.0+04$ & 5.6 & $2.06-04$ & 36.1 \\
\hline $\mathrm{F}$ & 0.835 & 5.0 & $1.64-04$ & 28.7 \\
\hline$K(t)$ & & $\Sigma$ & $4.78-05$ & 8.4 \\
\hline$t_{s}$ & 530.2 & 7.2 & $5.70-04$ & 100.0 \\
\hline
\end{tabular}




\section{V.A.5. GAP TEMPERATURE DROP: $\Delta \mathrm{t}_{\mathrm{g}}$}

The gap temperature drop is defined as the difference between the fuel surface temperature and the cladding inside surface temperature, i.e.,

$$
\Delta t_{g}=t_{s}-t_{j},
$$

and its relative variance is given by

$$
c_{\Delta t_{g}}^{2}=\left(\frac{t_{s}}{\Delta t_{g}}\right)^{2} c_{t_{s}}^{2}+\left(\frac{t_{i}}{\Delta t_{g}}\right)^{2} c_{t_{i}}^{2}-2 \frac{t_{j} t_{s}}{\Delta t_{g}{ }^{2}} c_{t_{j} t_{s}}
$$

where

$$
c_{t_{i} t_{s}}=\frac{-\frac{q F}{4 \pi}\left(\Delta t_{f}+\Delta t_{c}\right)}{t_{i} t_{s} K\left(t_{s}\right)} c_{q}^{2} .
$$

\begin{tabular}{|c|c|c|c|c|}
\hline \multirow[b]{2}{*}{ Parameter } & \multirow[b]{2}{*}{ Value } & \multirow{2}{*}{$\begin{array}{c}\text { Uncertainty, } \\
\%\end{array}$} & \multicolumn{2}{|c|}{ Contribution to $c_{t}^{2}$} \\
\hline & & & Value & $\%$ of Total \\
\hline$t_{s}$ & 530.2 & 7.2 & $3.03-03$ & 88.6 \\
\hline$t_{i}$ & 300.08 & 1.8 & $5.92-05$ & 1.7 \\
\hline$t_{i}, t_{s}$ & & & $3.33-04$ & 9.7 \\
\hline$\Delta t_{g}$ & 230.12 & 17.5 & $3.42-03$ & 100.0 \\
\hline
\end{tabular}

An example calculation is given in Table 8.

TABLE 8. Gap Temperature Drop Uncertainty: Example 


\section{V.B. CALCULATION OF UNCERTAINTY FOR GAP CONDUCTANCE}

The uncertainty calculation for the gap conductance are derived for two different situations. First, the error propagation method used for the intermediate parameters is derived for the case where it is reasonable to assume that the error distribution for the gap conductance is symmetric and approaches the Gaussian distribution, i.e., the assumptions at the beginning of Section $V$ hold. When this is not the case, e.g., for small gap temperature drops, an alternate error analysis is given. The method is not restricted to the nonsymmetric case. It can be used for the uncertainty calculations for gap conductance in all cases. If the gap conductance error distribution is symmetric, the uncertainty limits are symmetric. If the distribution is nonsymmetric, the limits are nonsymmetric.

The gap conductance is defined as

$$
h_{g}=\frac{q}{\pi_{d} \Delta t_{g}}
$$

where $d$ is the diameter selected for fuel-cladding gap. The relative variance for the gap conductance when derived by error propagation is

$$
c_{h_{g}}^{2}=c_{q}^{2}+c_{d}^{2}+c_{\Delta t_{g}}^{2}-2 c_{q \Delta t_{g}}+2 c_{d \Delta t_{q}}
$$

where

$$
c_{q \Delta t_{q}}=\frac{-\left(\frac{q F}{4 \pi k\left(t_{s}\right)}+\Delta t_{f}+\Delta t_{c}\right)}{\Delta t_{g}} c_{q}^{2}
$$

and when

$$
\begin{aligned}
d & =\left(d_{i}+d_{p}\right) / 2 \\
c_{d}^{2} & =\frac{d_{i}^{2} c_{d_{i}}^{2}+d_{p}^{2} c_{d_{p}}^{2}}{4 d^{2}}
\end{aligned}
$$




$$
c_{\Delta t_{g}, d}=\frac{1}{2} \frac{d_{i} \Delta t_{c}}{d \Delta t_{g}{ }_{n}\left(d_{0} / d_{j}\right)} c_{d_{i}}^{2}
$$

If the gap conductance is calculated utilizing only the inside cladding diameter, then $d=d_{i}$ and the relative variance may be obtained using the same equations but with the following two changes

$$
\begin{aligned}
c_{d}^{2} & =c_{d_{i}}^{2} \\
c_{\Delta t, d} & =\frac{\Delta t_{c}}{\Delta t \ln \left(d_{0} / d_{i}\right)} c_{d_{i}}^{2}
\end{aligned}
$$

An example application of the error propagation formulas is contained in

\begin{tabular}{|c|c|c|c|c|}
\hline \multirow[b]{2}{*}{ Parameter } & \multirow[b]{2}{*}{ Value } & \multirow[b]{2}{*}{$\begin{array}{c}\text { Uncertainty, } \\
\% \\
\end{array}$} & \multicolumn{2}{|c|}{ Contribution to $C_{h}^{2}$} \\
\hline & & & Value & $\%$ of Total \\
\hline$q$ & $3.0+04$ & 5.6 & $3.48-04$ & 6.7 \\
\hline$d$ & $1.087-02$ & 0.2 & $2.22-07$ & $<0.01$ \\
\hline$\Delta \mathrm{t}_{\mathrm{q}}$ & 230.12 & 17.5 & $3.42-03$ & 65.9 \\
\hline $\mathrm{q}, \Delta \mathrm{t}_{\mathrm{q}}$ & & & $1.42-03$ & 27.4 \\
\hline$d, \Delta t_{q}^{y}$, & & & $6.07-07$ & $<0.01$ \\
\hline$h_{g}\left(\stackrel{W}{\left.W / m^{2}-C\right)}\right.$ & $0.381+04$ & 21.6 & $5.19-03$ & 100.0 \\
\hline
\end{tabular}
Table 9.

TABLE 9. Gap Conductance Uncertainty: Symmetric Example 
The validity of the errors propagation method for determining the percent uncertainty for the gap conductance depends on a symmetry assumption for the gap conductance error distribution. In particular the distribution must resemble a Gaussian distribution. The previously described output parameters are generally expected to satisfy this assumption. However, the gap conductance is essentially the ratio of the two dependent parameters linear heat rating, $q$, and gap temperature drop, $\Delta \mathrm{t}_{\mathrm{g}}$. In this situation if the uncertainty for $\Delta \mathrm{t}_{\mathrm{g}}$ is reasonably large, then, along with the dependence, the distribution of $\mathrm{h}_{\mathrm{g}}$ is expected to be nonsymmetric with stretched tail for high gap conductances. The error propagation method of obtaining an uncertainty for gap conductance is not adequate in this situation.

An al ternate procedure based on the known dependence and assumed Gaussian distributions of the linear heat rating and gap temperature drop is available. The procedure, due to Fieller, ${ }^{(6)}$ gives nonsymmetric limits for the uncertainties. The method first calculates a confidence interval for the gap conductance. This interval is computed to correspond to the three sigma 1 imit chosen to represent the interpretation given to the uncertainties. This choice is reflected in the parameter $t$ given below. 
The computations for Fieller's method are given by

$$
h_{L}, h_{u}=h_{g} \frac{1-t^{2} c_{x_{y}} \mp t \sqrt{\text { DISCR }}}{1-t^{2} c_{x}^{2}}
$$

where

$$
\begin{aligned}
\text { DISCR } & =t^{2} c_{X_{y}}^{2}-2 c_{X_{Y}}+c_{y}^{2}+\left(1-t^{2} c_{y}^{2}\right) c_{X}^{2} \\
c_{x}^{2} & =c_{\Delta t_{g}}^{2}+c_{d}^{2}+2 c_{d \Delta t_{g}} \\
c_{y}^{2} & =c_{q}^{2} \\
c_{x} & =c_{q \Delta t_{g}} \\
t & =3.0 .
\end{aligned}
$$

The calculated gap conductance limits may be changed to percent uncertainties by

$$
\frac{h_{L}-h_{g}}{h_{g}} \times 100 \text { and } \frac{h_{u}-h_{g}}{h_{g}} \times 100
$$

In calculating the upper and lower limits two apparent anomalous situations can occur. First, the value of DISCR can be negative. This implies the limits are from 0 to infinity. Second, the computed interval may not include the $h_{g}$ value. In this case, the actual interval includes all real values outside of the calculated interval. Both of these situations are a consequence of large uncertainty associated with $\Delta t_{g}$. 
Table 10 shows the necessary information for calculating the uncertainty limits. This example is the same as for Tables 4-9. The uncertainty based on the error propagation is 21.6 percent.

TABLE 10. Gap Conductance Uncertainty: Nonsymmetric Example

\begin{tabular}{|c|c|c|c|}
\hline Parameter & Value & $\begin{array}{c}\text { Uncertainty, } \\
\frac{\%}{0}\end{array}$ & $\begin{array}{l}\text { Relative Variance } \\
\text { Covariance }\end{array}$ \\
\hline$q$ & $3.0+04$ & & $3.48-04$ \\
\hline$\Delta t_{a}$ & 230.12 & 17.5 & $3.42-03$ \\
\hline$d^{g}$ & $1.087-02$ & 0.2 & $4.44-07$ \\
\hline$q, \Delta t_{g}$ & & & $1.42-03$ \\
\hline$d, \Delta t_{g}$ & $0.381+04$ & $-18.4 \& 26.1$ & $6.07-07$ \\
\hline
\end{tabular}




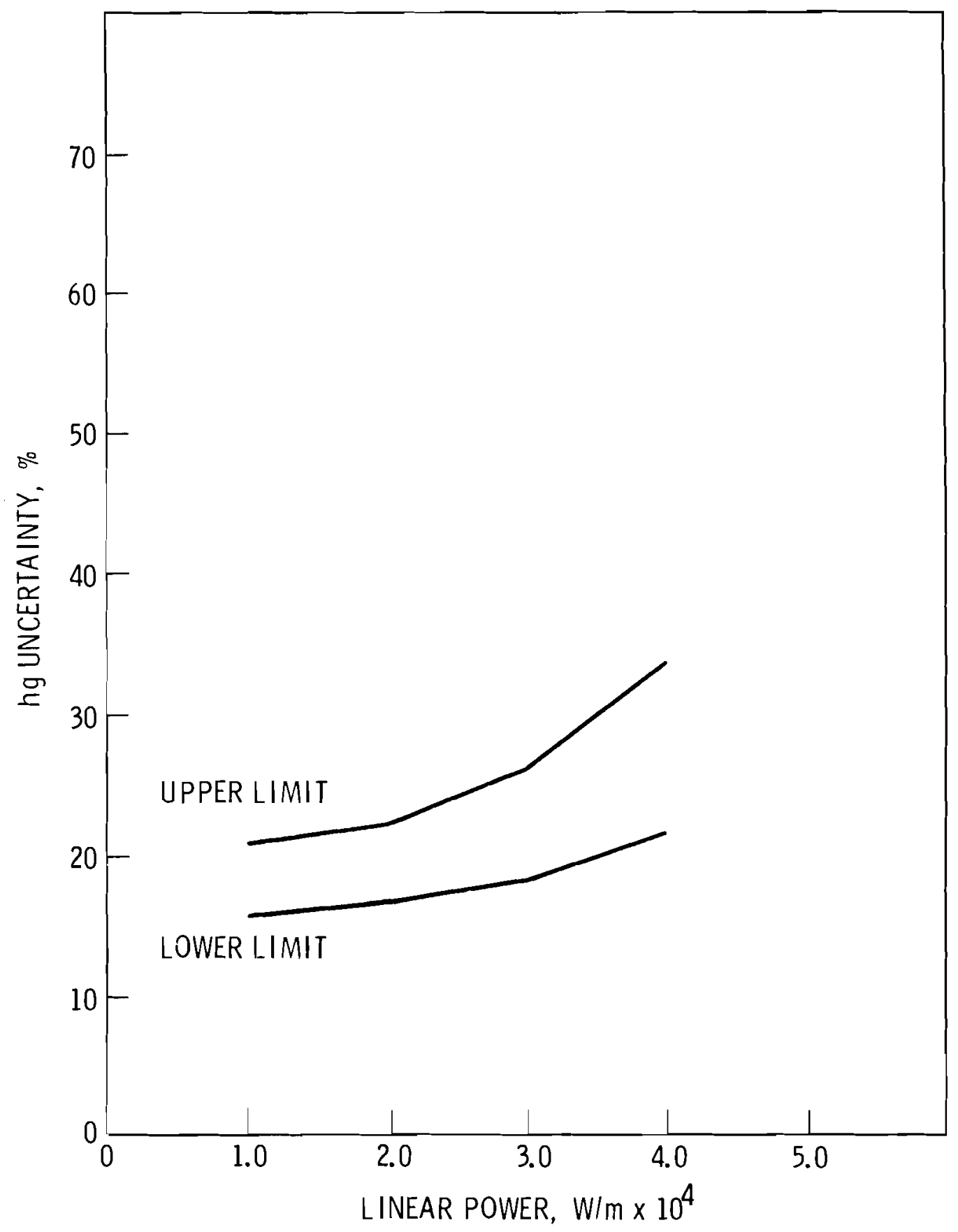

FIGURE 5. Gap Conductance Uncertainty Versus Linear Power for Rods 1, 5, and 6, IFA-431 
6

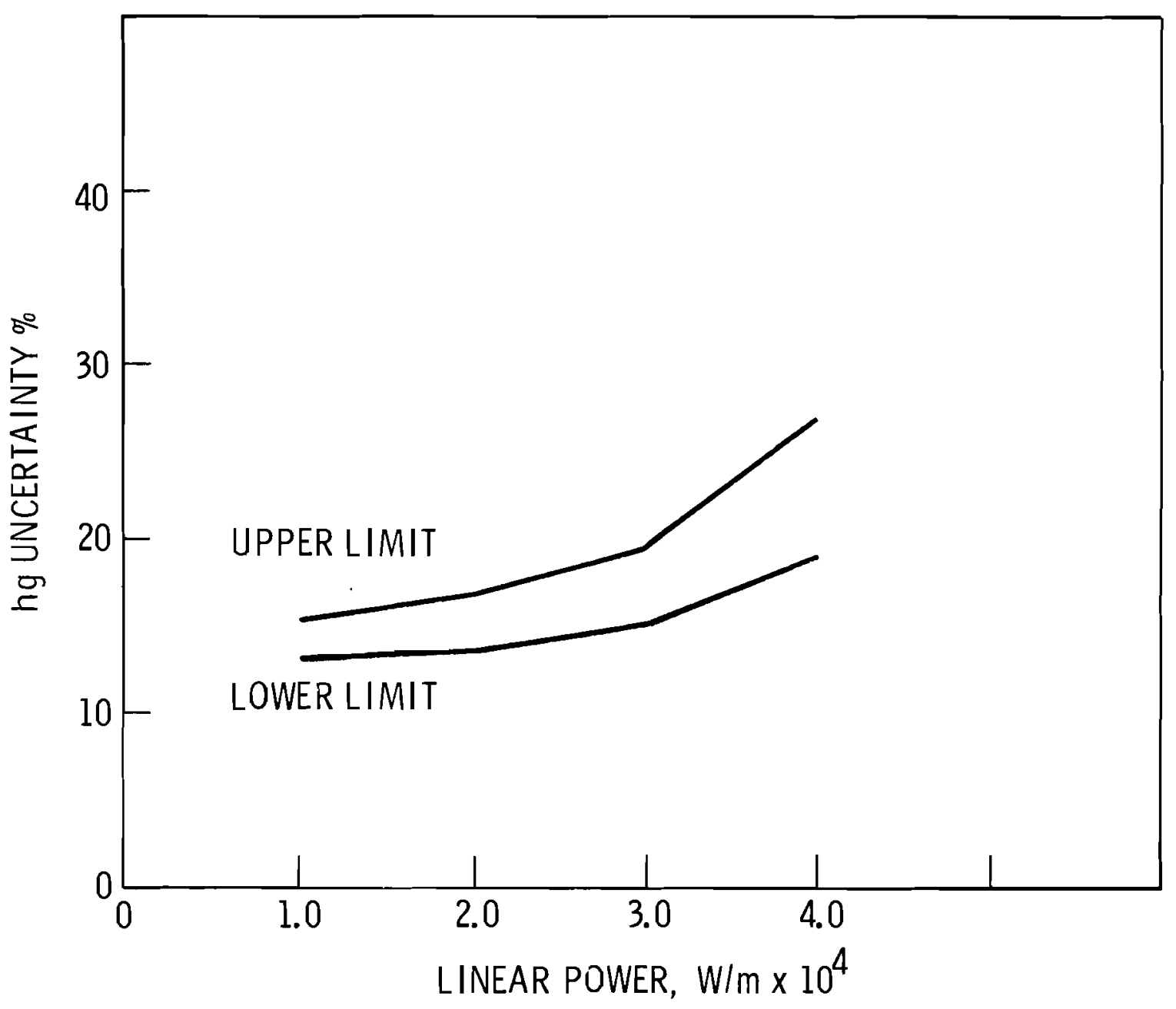

FIGURE 6. Gap Conductance Uncertainty Versus Linear Power for Rod 2, IFA-431 


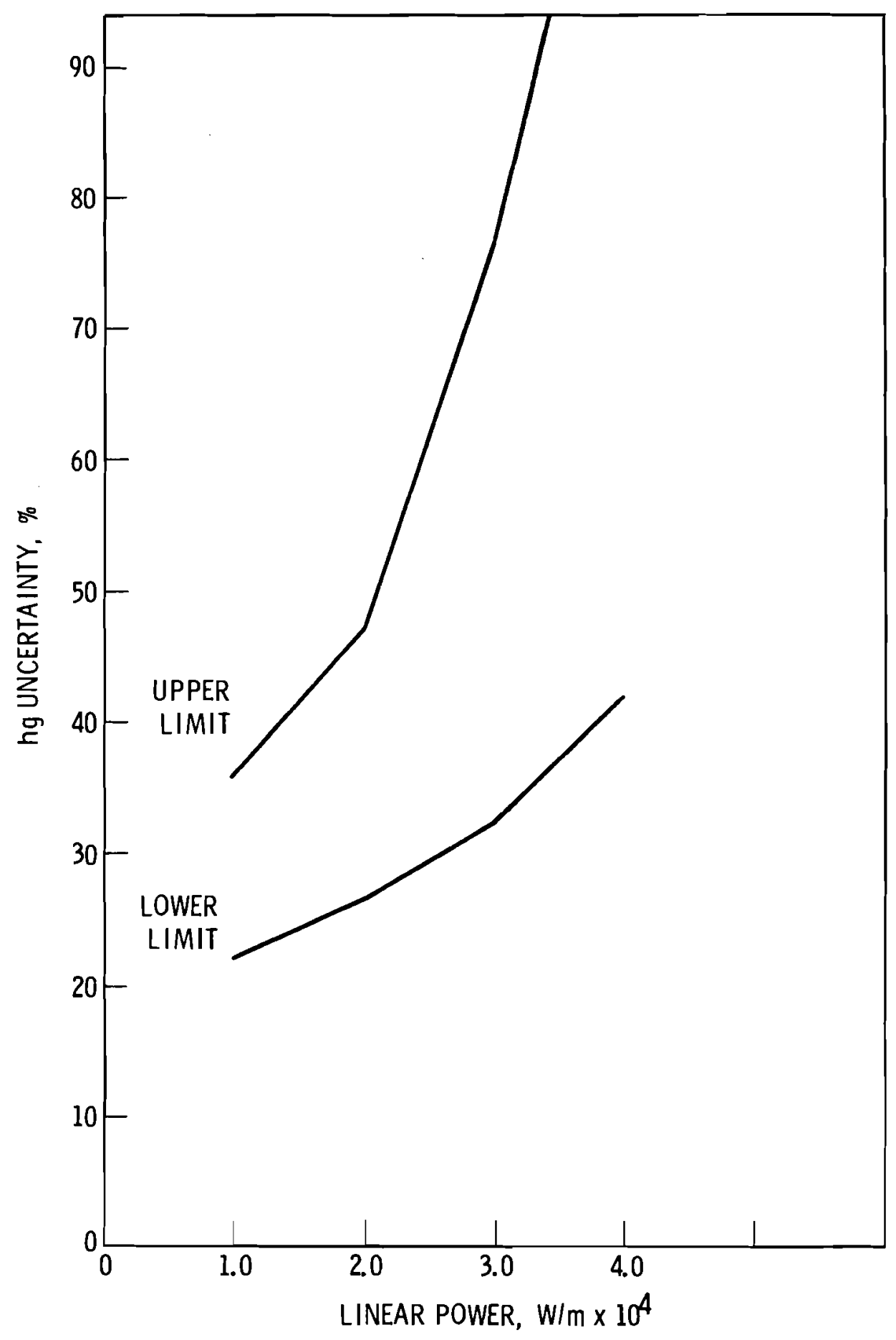

FIGURE 7. Gap Conductance Uncertainty Versus Linear Power for Rod 3, IFA-431 


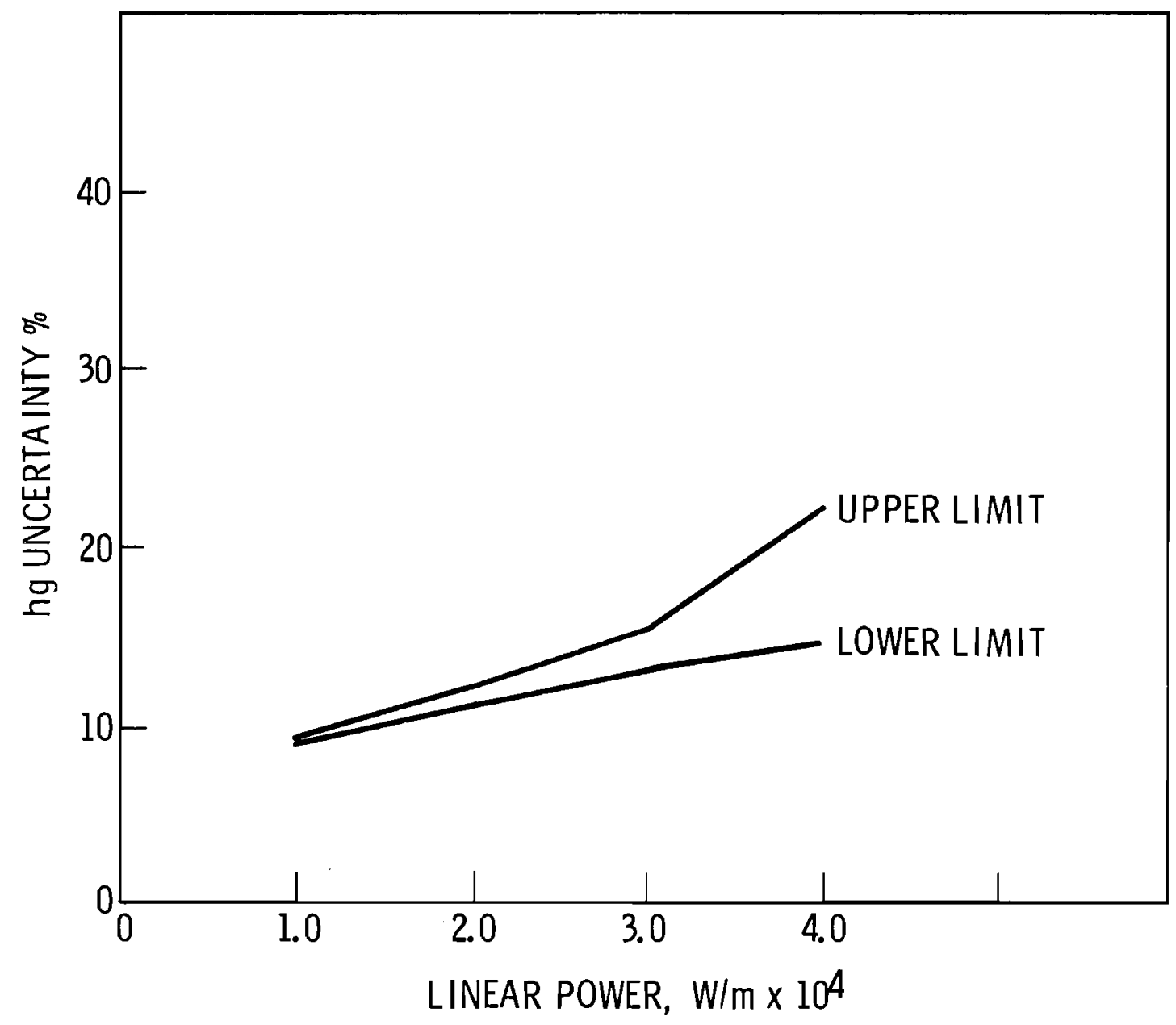

FIGURE 8. Gap Conductance Uncertainty Versus Linear Power for Rod 4, IFA-431 


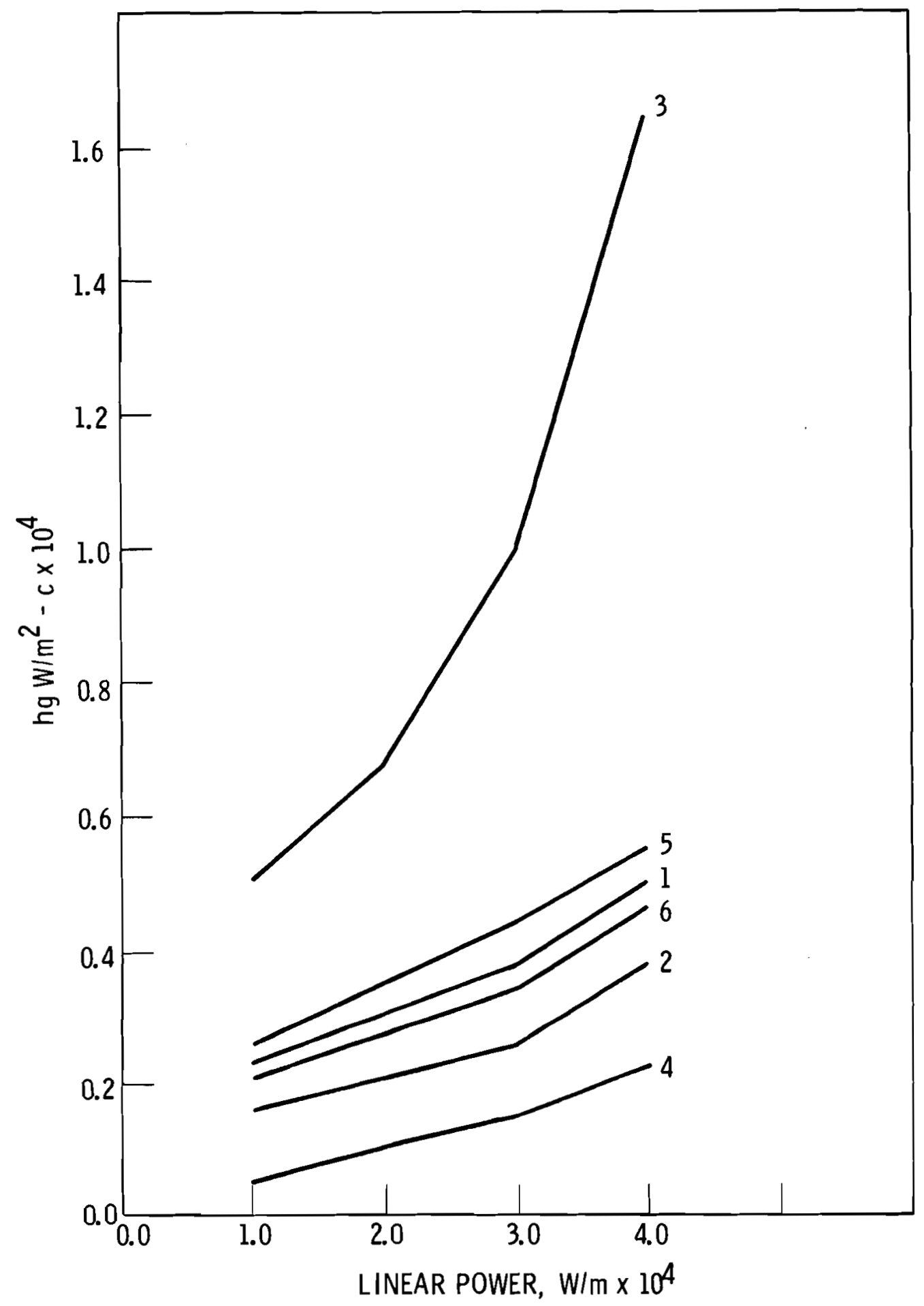

FIGURE 9. Gap Conductance Versus Linear Power for Rods 1-6, IFA-431 


\section{REFERENCES}

1. C. E. Beyer, C. R. Hann, D. D. Lanning, F. E. Panisko and L. J. Parchen, GAPCON-THERMAL-2: A Computer Program for Calculating the Thermal Behavior of an Oxide Fuel Rod. BNWL-1898, Battelle, Pacific Northwest Laboratories, Richland, WA 99352, November 1975.

2. J. A. Dearien, G. A. Berna, M. P. Bohn, D. R. Colemen, P. E. MacDonald and J. M. Broughton, FRAP-S: A Computer Code for the Steady-State Analysis of Oxide Fuel Rods. SRD-52-76, Aerojet Nuclear Company, INEL, Idaho Fal1s, ID 83401, January 1976.

3. J. A. L. Robertson, $\int K d \theta$ in Fuel Irradiations. CRFD-835, Chalk River, Ontario, Canada, Aprî 1959.

4. J. Jaech, Statistical Methods in Nuclear Material Control. TID-26298.

5. C. R. Hann et al., "Test Design, Precharacterization and Assembly Fabrication for Instrumented Fuel Assemblies." IFA-431 and IFA-432, BNWL-1933.

6. E. C. Fieller, "Some Problems in Interval Estimation," J. Royal Statistical Society, B. Vol 16, pp. 175-185, 1954.

7. Letter C. R. Hann to R. Lobel (USNRC-DOR) "Addendum to Report on $\mathrm{UO}_{2}$ Thermal Conductivity." June 25, 1976.

8. B. Aarset, Calibration Techniques and Data Handling Equipment Instrumented Fuel Assemblies in HBWR. HPR79, OECD Halden Reactor Project, Halden, Norway, December 1967.

9. M. Brakas, B. Söderlund and 0. Stringdehag, Long Term Test of SelfPowered Neutron Detectors in HBWR. HPR 154, OECD Halden Reactor Project, Halden, Norway, June 1972.

10. A. J. Walter, "Thermocouple Calibration: Irradiation arid Transmutation Effects." CONF-741226-1, Summary of information presented at the International Colloquium on High Temperature In-Pile Thermometry, Petten, Netherlands, December 1974.

11. C. L. Bennett and W. L. Purce11, BRT-I: Battelle-Revised-THERMOS BNWL-1434, Battelle, Northwest Laboratories, Richland, WA 99352, 1970.

12. F. Mathieu et al., "Thermoelectrically Generated Noise in Sheathed Thermocouples and in Other Low Level Instrumentation Cables." Annals of Nuclear Energy. 2: 127-140, 1975.

13. R. Isnard, "Heat Transfer Coefficient and Some Other Reactor Parameters Determined from Measurements of Fuel Temperature and Neutron Flux Fluctuations." Annals of Nuclear Energy. 2: 193-195, 1975. 
APPENDIX A

DISCUSSION OF ASSIGNED UNCERTAINTY VALUES 


\section{DISCUSSION OF ASSIGNED UNCERTAINTY VALUES}

This appendix discusses the factors involved in deducing each of the major input variables for $h_{\text {gap }}$.

\subsection{LINEAR HEAT RATING, q}

The linear heat rating is obtained from a combination of assembly power and neutron detector outputs. Each of these will be treated in turn.

\subsection{Fuel Assembly Power}

At the beginning of the first irradiation cycle, the total power in the test assembly is calibrated thermal-hydraulically under forced convection cooling and is related to the average of the neutron detector currents. After this calibration the assembly is operated under natural circulation cooling, and the assembly power and individual fuel rod linear heat ratings are deduced from the neutron detector currents. The calibration is not repeated.

The test assembly inlet ports near the bottom of the assembly as shown in Figure A-1 are closed for the forced convection calibration run by activating the calibration valve.

Forced circulation cooling is introduced from the subcooled plenum chamber and boiling is established in the channel. This is shown schematically in Figure $A-1$.

The assembly is operated at constant power which is controlled by the neutron detector readings while the subcooling is varied. The boiling power is computed and plotted versus the measured subcooled power. Figure A-2 shows a plot copied from digital computer results. The plots of boiling power versus subcooled power are very close to a straight line. The intersection of the extrapolated plots with the abscissa gives the minimum subcooled power required 


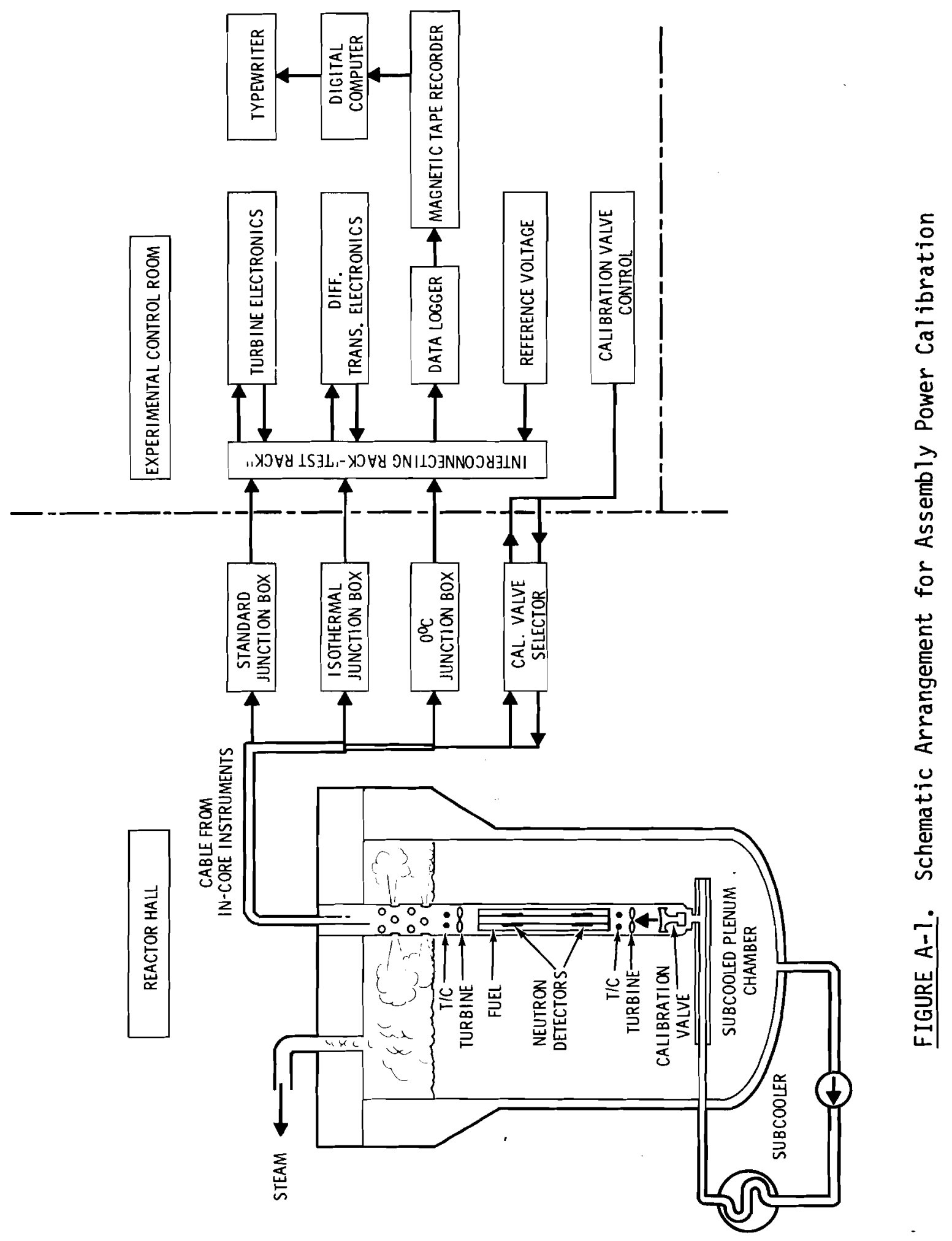




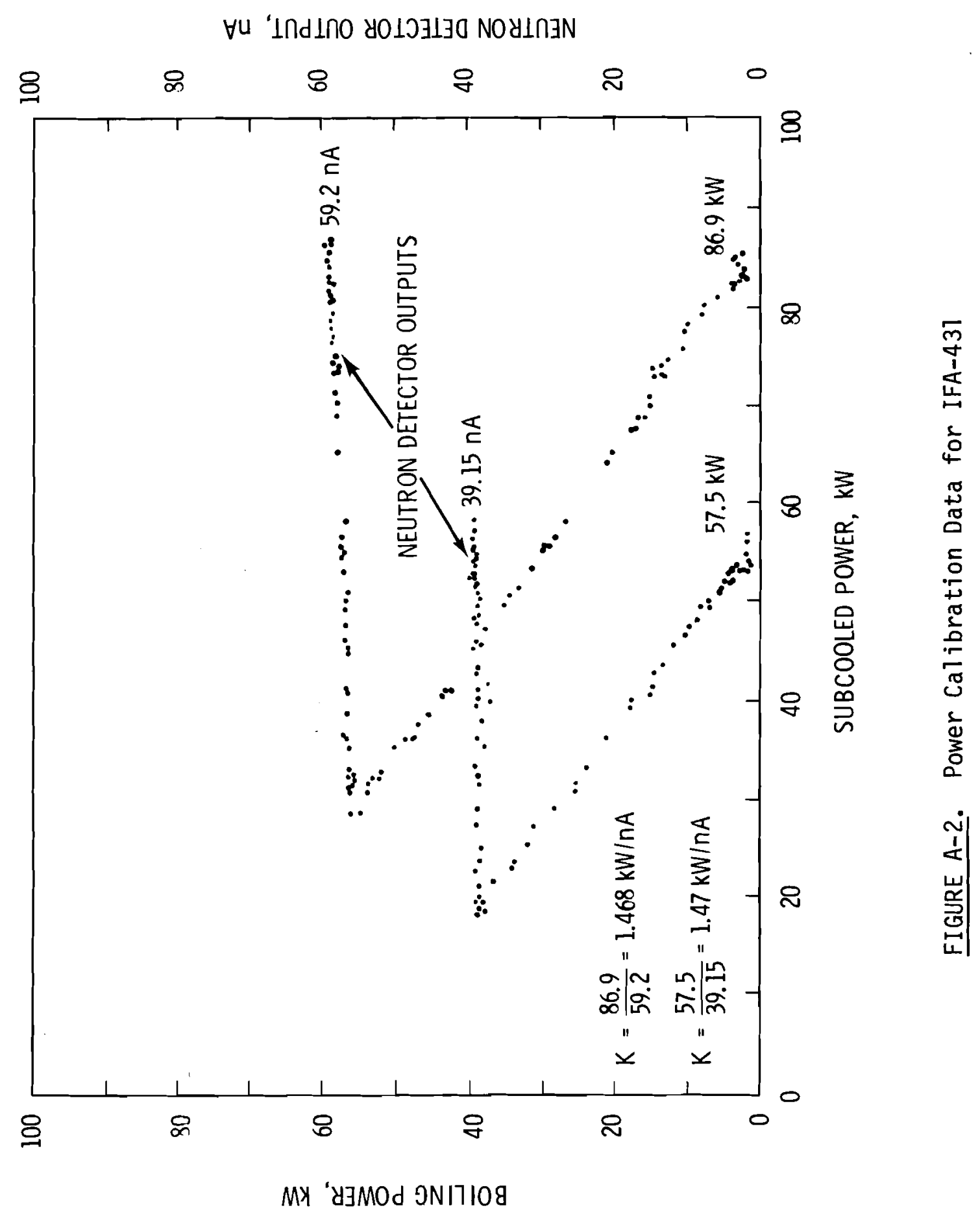


to suppress boiling in the channel under ideal conditions. The subcooled power is equal to the total channel power which is corrected for the moderator heating effect to give the channel power associated with the assembly.

The accuracy of the assembly power determination depends on the following factors: ${ }^{(8)}$

- calibration of inlet and outlet turbine flow meters

- calibration of coolant thermocouples

- temperature profile across the coolant channel where the inlet and outlet thermocouples are located

- influx of heat from the moderator into the coolant channel

- slip factor constancy

- signal to noise ratio

- accuracy of data handling equipment.

The outlet turbine accuracy is very good, better than $\pm 0.5 \%$, while the inlet turbine calibration is more doubtful due to the short and complicated inlet section. Comparison with the outlet turbine immediately prior to measurements gives good control, however, and the accuracy obtained is estimated to be better than $\pm 2 \%$.

The calibration of coolant thermocouples is very good, about $\pm 2 \mu \mathrm{V}$. An $18^{\circ} \mathrm{F}\left(10^{\circ} \mathrm{C}\right)$ temperature rise in the channel has an accuracy of $\pm 0.5 \%$.

The nonuniform temperature distribution across the inlet to the channel is negligible because there is excellent mixing before the coolant enters the assembly. The distribution across the outlet, however, is difficult to determine as there is little information available. The best estimate is obtained by studying the consistency of the measurements and the degree of agreement between the various calibration methods.

The influence of th. 3 heat flux from the moderator is small. It can amount to as much as $10 \mathrm{~kW}$ at low channel power and high subcooling, but it is taken into account, so even if some error is involved in determining this heat flux, the overal1 error contribution is very sma 11 , probably less than $\pm 0.5 \%$. 
The calibration method is based on assuming a constant slip factor during a subcooled power ramp. The results of the measurements give a plot of boiling power versus subcooled power which is very close to a straight line, indicating that the assumption is valid.

The signal to noise ratio does not normally cause any difficulty with sound instruments. The noise is easily filtered out.

There is always full control with the performance of the data handling system, so errors caused in this equipment can be neglected compared to error from other sources.

Another factor which influences the accuracy of assembly power indication by the beta current neutron detectors is the specificity of the power indicators themselves. Part of the neutron flux registered by these instruments originates in other fuel assemblies, and this portion of the signal will depend on the flux distribution in the core.

Taking the above factors into account, Halden estimates that the in-reactor assembly power measurements have accuracies in the \pm 3 to $\pm 5 \%$ range. (4)

A value of $\pm 41 / 2 \%$ was selected for the IFA-431 assembly power accuracy.

\subsection{Neutron Detector Output}

Each assembly is equipped with six vanadium, self-powered, beta current neutron detectors to monitor the power in the fuel assembly after the initial thermal-hydraulic calibration. One detector failed in the IFA-431 assembly shortly after startup.

Each vanadium neutron detector is $3.93 \mathrm{in} .(100 \mathrm{~mm})$ long and is located so that the center of the detector and the thermocouple junction are located on essentially the same plane.

The neutron detector output is an average of the current generated over the length of the detector. A nonsymmetrical flux gradient or spiked flux along the length of the detector could produce incorrect power generation results. It appears that the axial flux gradients along the neutron detectors are symmetrical and without spikes, so errors from this source should be minimal. 
The neutron detectors used in the test assemblies are not calibrated. Their precisions are based on the results of the irradiation of 30 similar type vanadium neutron detectors in the Studsvik R2-0 Reactor in Sweden. The 30 detectors were irradiated in a thermal neutron flux of $1.1 \times 10^{10} \mathrm{n} / \mathrm{cm}^{2}-\mathrm{sec}$. The error limits for the outputs of the detectors are estimated to be $\pm 2.5 \%$ at a neutron flux of $1.1 \times 10^{11} \mathrm{n} / \mathrm{cm}^{2}-\mathrm{sec}$.

In addition to correlating the detector outputs to the neutron flux in the Studsvik reactor, Halden has conducted long-term tests of similar neutron detectors in the HBWR. (9) These long-term tests have established the detectors as a reliable and accurate instrument without a measurable change in sensitivity at the higher flux levels in the HBWR.

The sensitivities of the test assembly neutron detectors are calculated from the sensitivities of the calibrated detectors and the physical characteristics of the test assembly detectors supplied by the manufacturer. To account for uncertainties in this calculation, $\pm 0.5 \%$ is added to the $\pm 2.5 \%$ for the calibrated detectors to give an uncertainty of $\pm 3.0 \%$ for the test assembly detectors.

The vanadium detectors have a calculated burnup rate of $0.013 \% /$ month at a neutron flux of $1 \times 10^{13} \mathrm{n} / \mathrm{cm}^{2}-\mathrm{sec}$. Based on this rate, the neutron detector end-of-1 ife (EOL) burnup for IFA-431 is $0.06 \%$. Because of this low value, the neutron detector outputs were not corrected for burnup.

A disadvantage of the vanadium detectors is their slow response time ( $5.5 \mathrm{~min}, 0$ to $63 \%$ ) to power changes. Consequently, during up and down power ramps, a correction factor should be considered for the output values. 


\subsection{Fuel Rod Linear Heating Rating}

The fuel rod linear heat rating is calculated from the following equation:

$$
q=\operatorname{CKNC}_{N}{ }^{*}
$$

where: $q=$ linear heat rating

$C=$ conversion factor which converts assembly power to individual rod linear heat rating

$K=$ calibration factor: ratio of assembly power to average of neutron detector currents

$\mathrm{N}=$ single neutron detector current

$C_{N}=$ neutron flux tilt correction factor.

The uncertainty associated with the conversion factor, $C$, takes into account the uncertainties in the dimensions, density, and enrichment of the fuel. This uncertainty is calculated to be $\pm 0.2 \%$. The uncertainty associated with $\mathrm{N}$ was evaluated as $\pm 3 \%$.

The IFA-431 test assembly is located in the outermost ring of the reactor where a significant radial flux tilt exists across the test assembly. This necessitates correcting the output signals from the neutron detectors to the axis of the fuel rods. This is accomplished with the flux tilt factor, $C_{N}$. We are assuming $\pm 1 \%$ uncertainty in the flux tilt factor.

The uncertainties associated with the calibration factor, $K$, and the linear heat rating, $q$, are developed in the following three paragraphs.

The available data for calculating the uncertainty for q consist of typical values for $C, K, N$, and $C_{N}$ along with uncertainty intervals for each factor where the errors are engineering estimates of error levels generally corresponding to maximum limits. The assumptions which are basic to this analysis are:

- The engineering estimates of the error limits are equivalent to three standard deviation 1 imits, i.e., $\pm 5 \%$ corresponds to $\pm\left(\frac{3 \sigma}{\mu}\right) 100$ where $\mu$ is the typical value and $\sigma$ is one standard deviation.

* A factor to correct for ${ }^{235} \mathrm{U}$ depletion and flux depression change should also be added. Since this factor is close to 1.0 for IFA-431 data and since its uncertainty is not easily estimated we have not included it in this report. 
- The four factors $C, K, N$ and $C_{N}$ are statistically independent. This assumption is reasonable since $K$ is determined at the initial startup and $\mathrm{N}$ is obtained from subsequent measurements. Furthermore, the variability in $C$ arises from measurement errors associated with the dimensions, density, and enrichment of the fuel, and $C_{N}$ is a flux tilt correction independent of neutron detector output.

By propagation of uncertainties, the 3 o uncertainty for the linear heat rating is

$$
U_{q}=\left(U_{C}^{2}+U_{K}^{2}+U_{N}^{2}+U_{C_{N}}^{2}\right)^{1 / 2}
$$

where $U_{C}$ is the engineering percent uncertainty on $C, U_{K}$ is the engineering percent uncertainty on $K, U_{N}$ is on $N$, and $U_{C_{N}}$ is on $C_{N}$. In order to utilize the above relationship, the 3 o percent uncertainty must be calculated for factor $k$.

The equation for calculating $K$ for IFA-431 is:

$$
K=\frac{Q_{C h}}{\left(N_{1}+N_{2}+N_{3}\right) / 6+\left(N_{4}+N_{5}\right) / 4}
$$

where $Q_{c h}$ is the calculated assembly power and $N_{j}$ is the output from the $i^{\text {th }}$ neutron detector. If it is assumed that the neutron detectors and the calculated assembly power are all statistically independent, then the above equation can be used along with propagation of errors to obtain the estimated error 1 imits for $K$. Utilizing these assumptions, the error limits for $K$ for IFA-431 are given by:

$$
U_{K}=\left(U_{c h}^{2}+\frac{5}{24} U_{N}^{2}\right)^{1 / 2}
$$

Because one detector failed in IFA-431, the neutron flux at the failed detector location is calculated from the operating detectors as follows:

$$
N_{6}=N_{3} \frac{\left[\left(N_{4} / N_{1}\right)+\left(N_{5} / N_{2}\right)\right]}{2}
$$


The uncertainty limits equation is

$$
U_{N_{6}}=\left\{U_{N_{3}}^{2}+\left(\frac{N_{3} N_{4}}{2 N_{1} N_{6}}\right)^{2}\left(U_{N_{1}}^{2}+U_{N_{4}}^{2}\right)+\left(\frac{N_{3} N_{5}}{2 N_{2} N_{6}}\right)^{2}\left(U_{N_{2}}^{2}+E_{N_{5}}^{2}\right)\right\}^{1 / 2}
$$

Detector No. 6 is located between Rods 4 and 5 at the upper thermocouple location. Consequently, the uncertainty of the linear heat rating for these two rods will be greater than that for the other rods.

The uncertainty assigned to the components of $q$ are listed below:

\section{TABLE A-1. Assigned Uncertainties for Linear Power Factors}

\begin{tabular}{lcc}
\multicolumn{1}{c}{ Item } & Uncertainty & Symbol \\
\cline { 2 - 3 } & $4.5 \%$ & $\mathrm{U}_{\mathrm{Ch}}$ \\
Detector Current & $3.0 \%$ & $\mathrm{U}_{\mathrm{N}}$ \\
Calibration Factor & $4.7 \%$ & $\mathrm{U}_{\mathrm{K}}$ \\
Flux Tilt Correction & $1.0 \%$ & $\mathrm{U}_{\mathrm{C}_{\mathrm{N}}}$ \\
Conversion Factor & (assumed) & ${ }_{\mathrm{U}}$
\end{tabular}

\subsection{CENTERL INE FUEL TEIPPERATURE}

Twelve thermocouples are used in IFA-431 for measuring the central fuel temperatures. The thermocouples have grounded junctions with $0.052 \mathrm{in}$. (1.575 mm) OD tungsten $22 \%$ rhenium sheaths and W $5 \%$ Re/W $26 \%$ Re seven-stranded thermocouple wires with beryllium oxide insulators.

The thermocouples were fabricated and calibrated by the Idaho National Engineering Laboratory (INEL). Calibration of the thermocouples over the range of use produces a brittle assembly which is fragile and subject to breakage. Consequently, only one thermocouple, which was not used in the in-reactor test, was calibrated.

The thermocouple was calibrated against a reference thermocouple of bare $\mathrm{W} 5 \% \operatorname{Re} / \mathrm{W} 26 \% \operatorname{Re}$ and an optical pyrometer as a second reference. The reference thermocouple and the optical pyrometer agreed within $40^{\circ} \mathrm{F}\left(22^{\circ} \mathrm{C}\right)$ up to $4000^{\circ} \mathrm{F}$ $\left(2204^{\circ} \mathrm{C}\right)$, but as the temperature approached $4500^{\circ} \mathrm{F}\left(2482^{\circ} \mathrm{C}\right)$, the two differed 
more widely. The optical pyrometer was thought to be closer since $4500^{\circ} \mathrm{F}$ temperature is above that given in most calibration tables for $W /$ Re thermocouples. The calibrated thermocouple had the following limits of error:

$$
\begin{aligned}
& \text { Ambient to } 1000^{\circ} \mathrm{F}\left(538^{\circ} \mathrm{C}\right) \\
& 1000^{\circ} \mathrm{F}\left(538^{\circ} \mathrm{C}\right) \text { to } 4000^{\circ} \mathrm{F}\left(2204^{\circ} \mathrm{C}\right)= \pm 10^{\circ} \mathrm{F}\left(5.5^{\circ} \mathrm{C}\right) \\
& 4000^{\circ} \mathrm{F}\left(2204^{\circ} \mathrm{C}\right) \text { to } 4500^{\circ} \mathrm{F}\left(2482^{\circ} \mathrm{C}\right)= \pm 2 \% \text { of reading }
\end{aligned}
$$

Irradiation of the thermocouples wil1 have long term effects caused by the shunting of the EMF's by conduction across the insulators, by transmutations in the thermocouple materials, and by temperature gradients along the thermocouple wires.

The insulator shunting effect was reduced to a negligible level by using beryllium oxide insulators.

The thermocouples used in these tests employ tungsten and rhenium which transmute under neutron irradiation to rhenium and osmium, respectively. These transmutations are the main cause of the reduction in the signal from the thermocouples with irradiation seen by investigators. (10) However, there is considerable variation in the magnitude of the effect seen. The reason for this variation is probably that the effect is not confined to changes occurring at the hot junction. The composition changes resulting from the transmutations give rise to inhomogeneities, causing Seebeck EMF generation in the wire situated within the temperature gradient. (12) The data from Reference 8 indicates an order of magnitude of about 10\% for the downward drift of the EMF at a thermal neutron fluence of $1 \times 10^{21} \mathrm{n} / \mathrm{cm}^{2}$.

The expected fluence for IFA-431 is about $1 \times 10^{20} \mathrm{n} / \mathrm{cm}^{2}-\mathrm{sec}$. Based on Reference 8, this fluence would indicate a downward EMF drift of 1\% for IFA-431 at EOL. No correction factor is applied to the IFA-431 data. However, an error value of $\pm 1 \%$ is assumed for the irradiation effects.

The average error band associated with the instrument measurement and conversion techniques over the range of temperature measurements is estimated to be $\pm 0.6 \%$. (9) 
Combining the calibration errors of $1 \%$ or $2 \%$, the irradiation effects error of $1 \%$, and the instrumentation of $0.6 \%$ and rounding off, gives the following estimated total uncertainties for the centerline fuel temperatures:

$1000^{\circ} \mathrm{F}\left(538^{\circ} \mathrm{C}\right)$ to $4000^{\circ} \mathrm{F}\left(2204^{\circ} \mathrm{C}\right)=\frac{\% \text { of Reading }}{ \pm 3}$
$4000^{\circ} \mathrm{F}\left(2204^{\circ} \mathrm{C}\right)$ to $4500^{\circ} \mathrm{F}\left(2482^{\circ} \mathrm{C}\right)= \pm 4$

\subsection{FUEL THERMAL CONDUCTIVITY}

The fuel thermal conductivities of the three fuel types employed in the test, 95\% TD stable, 92\% TD stable, and 92\% TD unstable, were determined. The method consists of heating one surface of a thin sample disc with a short heat pulse from a laser beam. The heat pulse passes through the sample, and the temperature transient on the back surface of the sample is measured and recorded. The thermal diffusivity is determined from the shape of the temperature-versustime curve.

The temperature transients were measured optically using a liquid nitrogen cooled, indium antimonide, infrared detector. The signal from the detector was displayed on an oscilloscope and recorded on film. Corrections were made for heat losses, but pulse time corrections were not required.

Each sample was initially heated to 1300 to $1400^{\circ} \mathrm{C}$ with measurements being taken at approximately $100^{\circ} \mathrm{C}$ intervals. On cooling, measurements were taken at 200 to $250^{\circ} \mathrm{C}$ intervals. Subsequently, each sample was heated to 1600 to $1650^{\circ} \mathrm{C}$. The samples were held at this temperature for 4 to 5.5 hours. Measurements were made to compare the thermal diffusivity before and after heat treatment. The sample was then cooled to room temperature and again heated to 1530 to $1650^{\circ} \mathrm{C}$ with measurements being made at 200 to $300^{\circ} \mathrm{C}$ increments.

The thermal diffusivity, $\alpha,\left(\mathrm{cm}^{2} / \mathrm{sec}\right)$ was calculated from the relationship.

$$
\alpha=\frac{t_{c} d^{2}}{t_{1 / 2}}
$$

where $t_{1 / 2}$ is the time for the back surface of the sample to reach $1 / 2$ the maximum temperature; $t_{c}$ is a heat loss correction which is determined from the shape of the time-temperature curve; and $d$ is the sample thickness. 
The thermal conductivity, $\lambda,(W / \mathrm{cm}-\mathrm{C})$ was calculated for each thermal diffusivity data point from the relationship

$$
\lambda=4.186 \alpha \cdot C_{p} \cdot p
$$

where $C_{p}\left(\mathrm{cal} / \mathrm{g}-{ }^{\circ} \mathrm{C}\right)$ is the heat capacity and $\left(\mathrm{g} / \mathrm{cm}^{3}\right)$ is the sample density.

The above limited amount of out-of-reactor thermal conductivity and temperature data were analyzed by a computer program which performs least squares solutions of linear equations by orthogonal transformations. The program generates coefficients for a third order polynomial, predicts a true value for the thermal conductivity at the input temperatures, and estimates a $95 \%$ upper and a 95\% lower limit. The thermal conductivity uncertainty intervals employed in this analysis are based on the limits estimated by the program for the outof-reactor data.

However, a curve fit to the expanded data base in Figure 4, which has more scatter but a higher density per temperature interval, shows similar results:

TABLE A-2. Comparison of Regression on Two Thermal Conductivity Data Sets

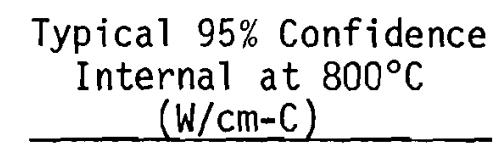

$\pm 7 \times 10^{-4}$

(Diffusivity Data

for IFA-431 Fuel)

Figure 4

(Expanded Data

Set per Ref. 6)

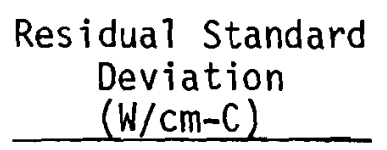

$\pm 2 \times 10^{-3}$

As noted in Appendix D, use of the curve fit to the expanded data set does not increase the $h_{\text {gap }}$ uncertainty very much. 


\subsection{THERMAL NEUTRON FLUX DEPRESSION}

The flux depression factor, $F$, was taken from the results of a THERMOS (17) calculation for a $10 \%$ enriched $\mathrm{UO}_{2}$ pellet. However, the uncertainty on $\mathrm{F}$ can be better appreciated by considering the approximate solution $I_{0}(K r)$ where $I_{0}$. is a modified Bessel's function and $K$ is the effective inverse diffusion length for the neutrons, and as such is proportional to the square root of the product of scattering and absorption cross sections for the fuel. With $F^{\prime}$ defined as

$$
\frac{2 \int_{0}^{a} \frac{d r}{r} \int_{0}^{r} r I_{0}(K r) d r}{\int_{0}^{a} r I_{0}(K r) d r}=F^{-}
$$

for a solid pellet, it is possible to define a range for $K$ such that $F^{-}=F \pm X \%$, for $F=0.85$. The following table summarizes the results.

TABLE A-3. Variation of $F$ With $K$

\begin{tabular}{ll}
\multicolumn{1}{c}{$\mathrm{F}^{-}$} & \multicolumn{1}{c}{$\mathrm{K}^{-}$} \\
\hline $0.9 \mathrm{~F}$ & $0.75 \mathrm{~K}$ \\
$0.95 \mathrm{~F}$ & $0.86 \mathrm{~K}$ \\
$0.98 \mathrm{~F}$ & $0.93 \mathrm{~K}$ \\
$1 . \mathrm{F}$ & $1 . \mathrm{K}$
\end{tabular}

From the table we see that a $\pm 5 \%$ variation in $F$ corresponds to a $\pm 14 \%$ variation in $\mathrm{K}$, which corresponds to about $\pm 20 \%$ variation in cross sections. 


\subsection{WATER FILM HEAT TRANSFER COEFFICIENT}

Experiments were performed in the SAPHIR swimming pool type reactor at the Institute for Reactor Research in würenlingen and in SILOETTE at the Center for Nuclear Studies in Grenoble to determine heat transfer coefficients. $(12,13)$

The average uncertainty for the SAPHIR data is $\pm 9.5 \%$ and that for SILOETTE is $\pm 15.1 \%$. The author did not give reasons for this difference nor were the reasons apparent from the data. A heat transfer coefficient calculated using Nusse1t, Reynolds, and Prandtl Numbers was shown by the author to lie within the range of uncertainty.

An uncertainty value of $\pm 15 \%$ for the water film heat transfer coefficient was selected.

\subsection{ADDITIONAL UNCERTAINTIES}

Additional uncertainties considered are those associated with crud deposition on the cladding surface, bulk water temperature, and cladding thermal conductivity. The uncertainties for the latter two parameters are as follows:

\section{Bulk water temperature \\ Cladding thermal conductivity} \pm Uncertainty

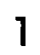

5

Crud deposition on fuel rods irradiated in the HBWR has rarely been observed during postirradiation examination. Crud was found on two assemblies which had a square lattice of fuel rods in a square shroud and on a burnout test which was taken into critical heat flux conditions numerous times. On fuel rods irradiated in assemblies with circular shrouds (IFA-431 and IFA-432 are in circular shrouds), adherent crud deposits have been virtually absent even to burnups in the 30,000 MWd/MTM range. (a) In view of this history of crud deposition in the HBWR, we are assuming crud-free surfaces during the irradiation. If postirradiation examination shows significant amounts of crud deposition, appropriate correction factors will be applied to the data.

(a) This information is taken from a memo dated December 16, 1975 - Crud Formation on Fuel Rods in HBWR - from Hanevik (Halden Reactor Project) to J. A. Christensen. 
APPENDIX B

EXAMPLES OF RELATIVE VARIANCE CALCILLATIONS 
A. Relative Variance of $\Delta t_{c}$

$$
\begin{aligned}
& \Delta t_{c}=\frac{q \ln \left(d_{0} / d_{i}\right)}{2 \pi b} \\
& \operatorname{Var}\left(\Delta t_{c}\right) \simeq\left(\frac{\partial \Delta t_{c}}{\partial q}\right)^{2} \sigma_{q}^{2}+\left(\frac{\partial \Delta t_{c}}{\partial d_{0}}\right)^{2} \sigma_{d_{0}}^{2}+\left(\frac{\partial \Delta t_{c}}{\Delta d_{i}}\right)^{2} \sigma_{d_{i}}^{2}+\left(\frac{\partial \Delta t_{c}}{\partial b}\right)^{2} \sigma_{b}^{2} \\
& \frac{\partial \Delta t_{c}}{\partial q}=\frac{\ln \left(d_{0} / d_{i}\right)}{2 \pi b}=\frac{\Delta t_{c}}{q} \\
& \frac{\partial \Delta t_{c}}{\Delta d_{0}}=\frac{q}{2 \pi b d_{0}}=\frac{\Delta t_{c}}{d_{0} \ln \left(d_{0} / d_{i}\right)} \\
& \frac{\partial \Delta t_{c}}{\partial d_{i}}=\frac{-q}{2 \pi t d_{i}}=\frac{-\Delta t_{c}}{d_{i} \ln \left(d_{0} / d_{i}\right)} \\
& \frac{\partial \Delta t_{c}}{\partial b}=\frac{-q \ln \left(d_{0} / d_{i}\right)}{2 \pi b^{2}}=\frac{-\Delta t_{c}}{b} \\
& c_{\Delta t_{c}}^{2}=\frac{\operatorname{var}\left(\Delta t_{c}\right)}{\left(\Delta t_{c}\right)^{2}} \simeq c_{q}^{2}+\frac{c_{d_{0}}}{\left[\ln \left(d_{0} / d_{i}\right)\right]^{2}}+c_{b}^{2}
\end{aligned}
$$


B. Relative Covariance of $\Delta t_{f}$ and $\Delta t_{c}, c_{\Delta t_{f}, \Delta t_{c}}$

$$
\begin{aligned}
\Delta t_{f}=\frac{q}{\pi d_{0} h_{f}} & \Delta t_{c}=\frac{q l n\left(d_{o} / d_{i}\right)}{2 \pi b} \\
\operatorname{Cov}\left(\Delta t_{c}, \Delta t_{f}\right) & \simeq\left(\frac{\partial t_{c}}{\partial q}\right)\left(\frac{\partial \Delta t_{f}}{\partial q}\right) \sigma_{q}^{2}+\left(\frac{\partial \Delta t_{c}}{\partial d_{0}}\right)\left(\frac{\partial \Delta t_{f}}{\partial d_{0}}\right) \sigma_{d_{0}}^{2} \\
& +\left(\frac{\partial \Delta t_{c}}{\partial h_{f}}\right)\left(\frac{\partial \Delta t_{f}}{\partial h_{f}}\right) \sigma_{h_{f}}^{2}+\left(\frac{\partial \Delta t_{c}}{\partial d_{i}}\right)\left(\frac{\partial \Delta t_{f}}{\partial d_{i}}\right) \sigma_{d_{i}}^{2} \\
& +\left(\frac{\partial \Delta t_{c}}{\partial b}\right)\left(\frac{\partial \Delta t_{f}}{\partial b}\right) \sigma_{b}^{2}
\end{aligned}
$$

First note that $\frac{\partial \Delta t_{c}}{\partial h_{f}}=\frac{\partial \Delta t_{f}}{\partial b}=\frac{\partial \Delta t_{f}}{\partial d_{i}}=0$ so that the last three terms are 0 . Then

$$
\begin{array}{ll}
\frac{\partial \Delta t_{c}}{\partial q}=\frac{\ln \left(d_{0} / d_{i}\right)}{2 \pi b}=\frac{\Delta t_{c}}{q} & \frac{\partial \Delta t_{f}}{\partial q}=\frac{\Delta t_{f}}{q} \\
\frac{\partial \Delta t_{c}}{\partial d_{0}}=\frac{\Delta t_{c}}{d_{0} \ln \left(d_{0} / d_{j}\right)} & \frac{\partial \Delta t_{f}}{\partial d_{0}}=\frac{-\Delta t_{f}}{d_{0}}
\end{array}
$$

Hence

$$
c_{\Delta t_{c}, \Delta t_{f}}=\frac{\operatorname{Cov}\left(\Delta t_{c}, \Delta t_{f}\right)}{\Delta t_{c} \Delta t_{f}}=c_{q}^{2}-\frac{c_{d_{0}}^{2}}{\ln \left(d_{o} / d_{i}\right)}
$$


APPENDIX C

MONTE CARLO SAMPLING 
- When the gap conductance distribution is approximately Gaussian, the error propagation uncertainty is adequate.

These conclusions are based on examining the Monte Carlo results using the IFA-431 rods 1 to 4 operating parameter values. (Only the general parameter values from the actual test data were used--not the actual history of the rods.) For each rod linear heat rating values were selected at 100, 200, 300 and $400 \mathrm{~W} / \mathrm{cm}$. Hence, the study consists of 16 different rod operating conditions. For each of the operating conditions the procedure was:

- To assume each input parameter was gaussian with mean and uncertainty specified by the operating conditions.

- To randomly generate 500 values for each input parameter.

- To perform the calculations necessary to obtain the intermediate parameters and gap conductance (completed 500 times).

- To evaluate for each input and output parameter the distribution of the 500 values (histogram, stem/leaf displays, probability plots, test statistics, etc.).

- To calculate the output parameters' average value and uncertainty for comparison against the methods in Section $V$.

The distributions of the input and intermediate parameters under al1 16 conditions appeared Gaussian when assessed by probability plotting and by the W-test for normality. In the case of gap conductance the distributions tended to be skewed to the right especially for rod 3 . Figure $\mathrm{C}-1$ shows a frequency plot of gap conductance values for the 500-element "rod 3 " sample at $3.0 \times 10^{4} \mathrm{~W} / \mathrm{m}$. The intervales are $0.02 \mathrm{~W} / \mathrm{m}^{2}-\mathrm{C} \times 10^{4}$, and the numbers within the plot represent the second digit to the right of the decimal place. Note the long tail at high $h_{g}$ values.

Comparisons of the error propagation analysis and the Monte Carlo results are contained in Tables $\mathrm{C}-1, \mathrm{C}-2$, and $\mathrm{C}-3$. The first two tables are for the intermediate parameters and the third for gap conductance. The agreement shown between the simulated and error propagation results is good for the 


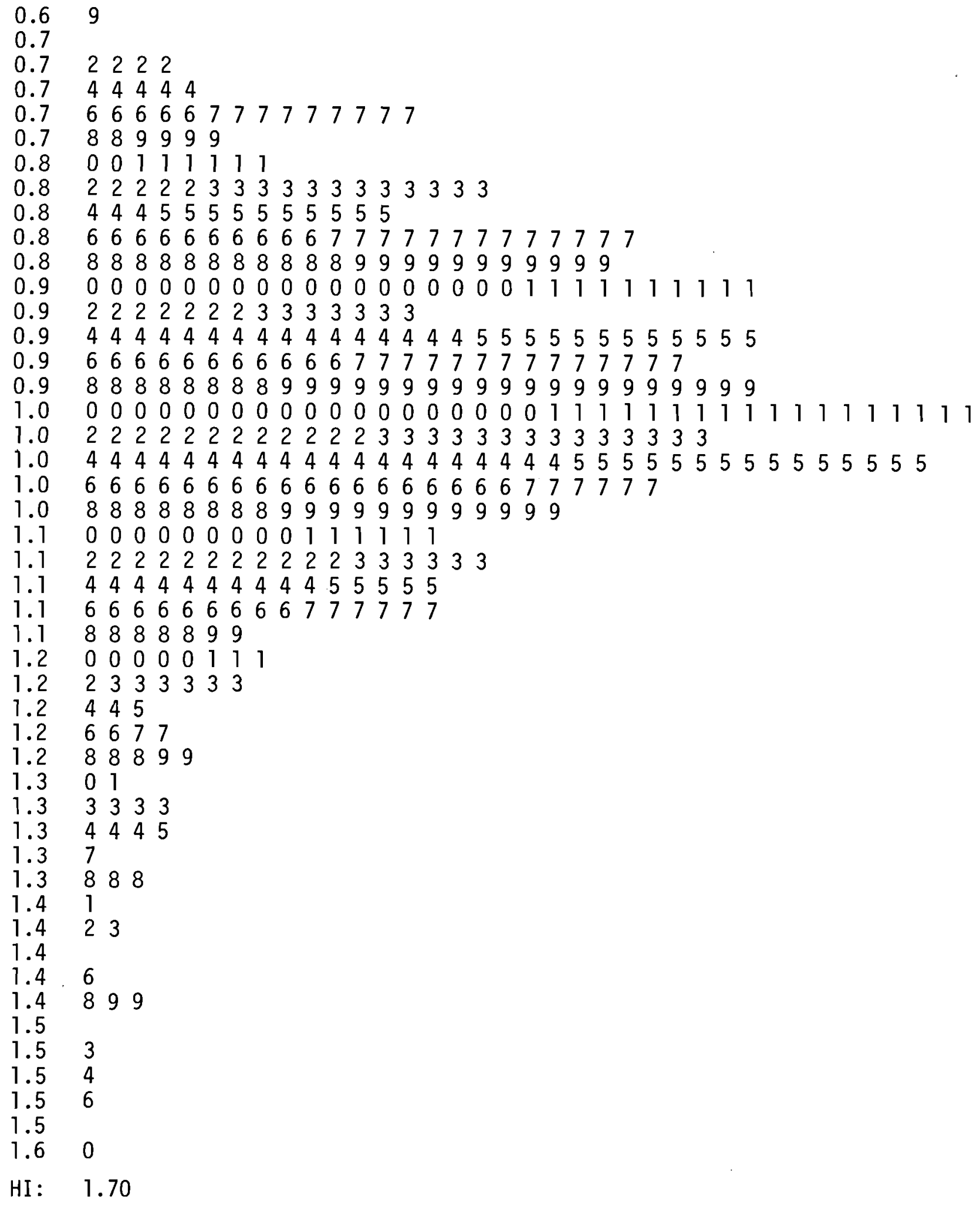

FIGURE C.I Stem/Leaf of Simulated Gap Conductance Values for $\operatorname{Rod} 3,3.0 \mathrm{~W} / \mathrm{m} \times 10^{4}$ 
TABLE C. 1 Comparison of Error Analys is and Monte Carlo Results for IFA-431 Rod 1 Intermediate Parameters

Parameter Error Analysis Monte Carlo

$\frac{\text { Uncertainty }}{\text { Error }}$
Analysis Monte Carlo

Film Temperature Drop $\Delta t_{f}$

$$
\begin{aligned}
& 1.0 \times 10^{4} \mathrm{~W} / \mathrm{m} \\
& 2.0 \\
& 3.0 \\
& 4.0
\end{aligned}
$$

4.2

8.5

12.7

16.9

4.2

8.5

12.7

17.0

Caldding Temperature Drop $\Delta t_{c}$

$$
\begin{aligned}
& 1.0 \times 10^{4} \mathrm{~W} / \mathrm{m} \\
& 2.0 \\
& 3.0 \\
& 4.0
\end{aligned}
$$

16.7

33.2

49.4

65.3
16.2

32.4

48.6

64.8
16.0

16.0

16.0

16.0

15.8

16.2

15.3

16.3
Inside Cladding Temperature $t_{i}$

$$
\begin{aligned}
& 1.0 \times 10^{4} \mathrm{~W} / \mathrm{m} \\
& 2.0 \\
& 3.0 \\
& 4.0
\end{aligned}
$$

258.9

279.7

300.1

320.2

258.4

278.9

299.3

319.8

7.5
7.5
7.5
7.5

7.7

7.1

7.7

7.5

7.2

Fuel Surface Density $t_{S}$

$$
\begin{aligned}
& 1.0 \times 10^{4} \mathrm{~W} / \mathrm{m} \\
& 2.0 \\
& 3.0
\end{aligned}
$$

375.3

467.6

530.2

558.0

375.5

467.7

530.3

557.8

$\begin{array}{ll}1.1 & 1.1 \\ 1.4 & 1.4 \\ 1.7 & 1.8 \\ 2.1 & 1.9\end{array}$

Del ta Temperature Drop $\Delta \mathrm{t}_{\mathrm{g}}$

$$
\begin{aligned}
& 1.0 \times 10^{4} \mathrm{~W} / \mathrm{m} \\
& 2.0 \\
& 3.0 \\
& 4.0
\end{aligned}
$$

116.4

187.9

230.1

237.8

117.1

188.7

231.0

238.0
4.4

5.6

7.4

9.0
8.8

4.3

5.8

8.8 
TABLE C.2 Comparison of Error Analysis and Monte Carlo Results for IFA-431 Rod 3 Intermediate Parameters

Parameter

Parameter Value Error Analysis Monte Carlo
Uncertainty

Error
Analysis Monte Carlo

Film Temperature Drop $\Delta t_{f}$

$$
\begin{aligned}
& 2.0 \times 10^{4} \mathrm{~W} / \mathrm{m} \\
& 3.0 \\
& 4.0
\end{aligned}
$$

8.5

12.7

16.9

33.2

49.4

65.3

32.4
48.7
64.8

7.5

7.5

7.5

7.6

7.0

7.9
16.9

16.1

Inside Cladding Temperature $t_{i}$

$$
\begin{aligned}
& 2.0 \times 10^{4} \mathrm{~W} / \mathrm{m} \\
& 3.0 \\
& 4.0
\end{aligned}
$$

Fuel Surface Temperature $t_{s}$

$$
\begin{aligned}
& 2.0 \times 10^{4} \mathrm{~W} / \mathrm{m} \\
& 3.0 \\
& 4.0
\end{aligned}
$$

Gap Temperature Gradient $\Delta \mathrm{t}_{\mathrm{g}}$
87.3

80.0

70.9
280.0

300.1

320.2

278.9

299.4

319.3

366.6

386.3

391.1

387.1

391.1

6.5

8.5

10.6

6.4

8.6

10.7
1.4

1.6

2.1

$$
\begin{aligned}
& 2.0 \times 10^{4} \mathrm{~W} / \mathrm{m} \\
& 3.0 \\
& 4.0
\end{aligned}
$$

87.7

86.9

72.1

29.0

28.1

40.6

63.4

40.5

63.3 
TABLE C.3 Comparison of Gap Conductance Error Analys is and Monte Carlo Results for IFA-431 Rods 1 and 3

$\frac{\text { Linear Heat Rating }(\mathrm{W} / \mathrm{m}) \times 10^{4}}{2.0}-\frac{4.0}{3.0}-1$

Rod 1

$\mathrm{hg}\left(\mathrm{W} / \mathrm{m}^{2}-\mathrm{C}\right) \times 10^{4}$

Error Analysis

Monte Carlo

0.32

0.38

0.50

0.31

0.38

0.50

Uncertainty (symmetric)

Error Analysis

Monte Carlo

Uncertainty (Fieller's)

Error Analysis

Monte Carlo

Rod 3

$$
\begin{aligned}
& \text { hg }\left(w / m^{2}-C\right) \times 10^{4} \\
& \text { Error Analysis } \\
& \text { Monte Carlo }
\end{aligned}
$$

Uncertainty (symmetric)

Error Analysis

Monte Carlo

Uncertainty (Fieller's)

Error Analys is

Monte Carlo
0.68
1.01
1.04
1.67
1.73

$\pm 21.6$

$\pm 26.2$

$\pm 18.6$

$\pm 26.8$

$-16.5,+22.2 \quad-18.4,+26.1-21.5,+33.6$

$-16.3,+21.8 \quad-19.1,+27.4 \quad-21.8,+34.3$ $\pm 32.7$

$\pm 33.2$

$-25.4,+45.9$

$-25.1,+44.4$ $\pm 44.5$

$\pm 47.4$

$\pm 67.4$

$\pm 79.9$ 
intermediate parameters. For gap conductance the uncertainty limits based on Fieller's method show good agreement. Fieller's method was applied to the simulated mean values and uncertainties for the linear heat rating and gap temperature drop in the same manner as presented in Section V.C. The error propagation uncertainties do not agree as well with the simulated results, especially as the uncertainty increases.

A direct comparison of the calculations for the gap conductance uncertainties with the simulated gap conductance values is presented in Table C-4. In this table the actual gap conductance limits are given for rods 1 and 3 based on both error propagation and Fieller's method. Note that the lower and upper limits are larger for Fieller's method. This is most acute for high uncertainties (as measured by error propagation). Two reasons for this shift can be given. First, the upper limit from Fieller's method takes account of the skewness in the gap conductance distribution. Second, because of the skewness the uncertainty estimate from the error propagation is inflated resulting in the limit being lower than necessary. The percent below and above items give the percentage of the 500 Monte Carlo values that are below and above the indicated limits. Based on the interpretation of uncertainty given in Section $V$, approximately $0.125 \%$ would be expected in each case, i.e., 0.6 observations below and above. Fieller's method satisfies this interpretation better than error propagation. 
TABLE C.4 Error Propagation and Fieller's Method Gap Conductance Limits and Comparison to Monte Carlo Distributions

(hg Values in $\mathrm{W} / \mathrm{m}^{2}-\mathrm{C} \times 10^{4}$ )

\begin{tabular}{lll} 
Linear Heat Rating $(\mathrm{W} / \mathrm{m}) \times 10^{4}$ \\
\hline 1.0 & 2.0 & 3.0
\end{tabular}

Rod 1

Error Propagation

$\begin{array}{lllll}\text { hg Lower Limit } & 0.205 & 0.260 & 0.298 & 0.369 \\ \text { Upper Limit } & 0.295 & 0.380 & 0.462 & 0.631 \\ \text { \% Below } & 0.0 & 0.2 & 0.0 & 0.0 \\ \% \text { Above } & 0.2 & 0.2 & 1.0 & 0.4\end{array}$

Fieller's Method

$\begin{array}{lllll}\text { hg Lower Limit } & 0.211 & 0.267 & 0.310 & 0.391 \\ \text { Upper Limit } & 0.302 & 0.391 & 0.479 & 0.668 \\ \text { \% Below } & 0.2 & 0.8 & 0.2 & 0.0 \\ \% \text { Above } & 0.0 & 0.0 & 0.2 & 0.0\end{array}$

Rod 3

Error Propagation

hg Lower Limit

Upper Limit

0.472

0.458

0.561

0.544

1.748

0.902

1.459

2.796

$\%$ Below

0.0

0.0

0.0

0.0

$\%$ Above

1.8

0.6

1.4

2.6

Fieller's Method

Lower Limit

Upper Limit

0.696

0.507

0.992

0.690

1.764

0.980

2.513

0.2

0.0

4.743

$\%$ Below

0.6

0.4

0.4

0.2

0.0

0.0 
APPENDIX D

PARAMETRIC STUDIES 


\section{PARAMETRIC STUDIES}

The four input variables which have the most effect on the uncertainty of deduced gap conductance are linear heat rating, fuel thermal conductivity, centerline temperature and flux depression. The assigned uncertainties for these input variables are subject to debate. We attempt to show in this appendix the effect of varying the assigned uncertainty of each of the first three parameters. The effect of variation of flux depression uncertainty would be similar to that for linear power.

1. The Linear Power, $q$

The assigned uncertainty for $q$ in this test was $\pm 5.6 \%$ relative. A more generally used estimate is $\pm 10 \%$ : Figure $D-1$ shows the uncertainty in $\mathrm{hg}$ as a function of $\Delta \mathrm{t}_{\mathrm{g}}$ at $300 \mathrm{~W} / \mathrm{cm}$ for both cases.

Note that for $U q=10 \%$, and $\Delta T_{\text {gap }}$ greater than $\sim 200 \mathrm{C}$, this figure implies that uncertainty in calculated fuel temperatures is always greater than $100 \mathrm{C}$ for powers equal to or greater than $300 \mathrm{~W} / \mathrm{cm}$.

2. The Centerline Temperature $t_{c}$

Increasing the uncertainty on the measured centerline temperature will also increase the uncertainty of hq, but not nearly so drastically as with q. Figure D-2 shows the result of doubling the assigned center1 ine temperature uncertainty of $\pm 3 \%$. 


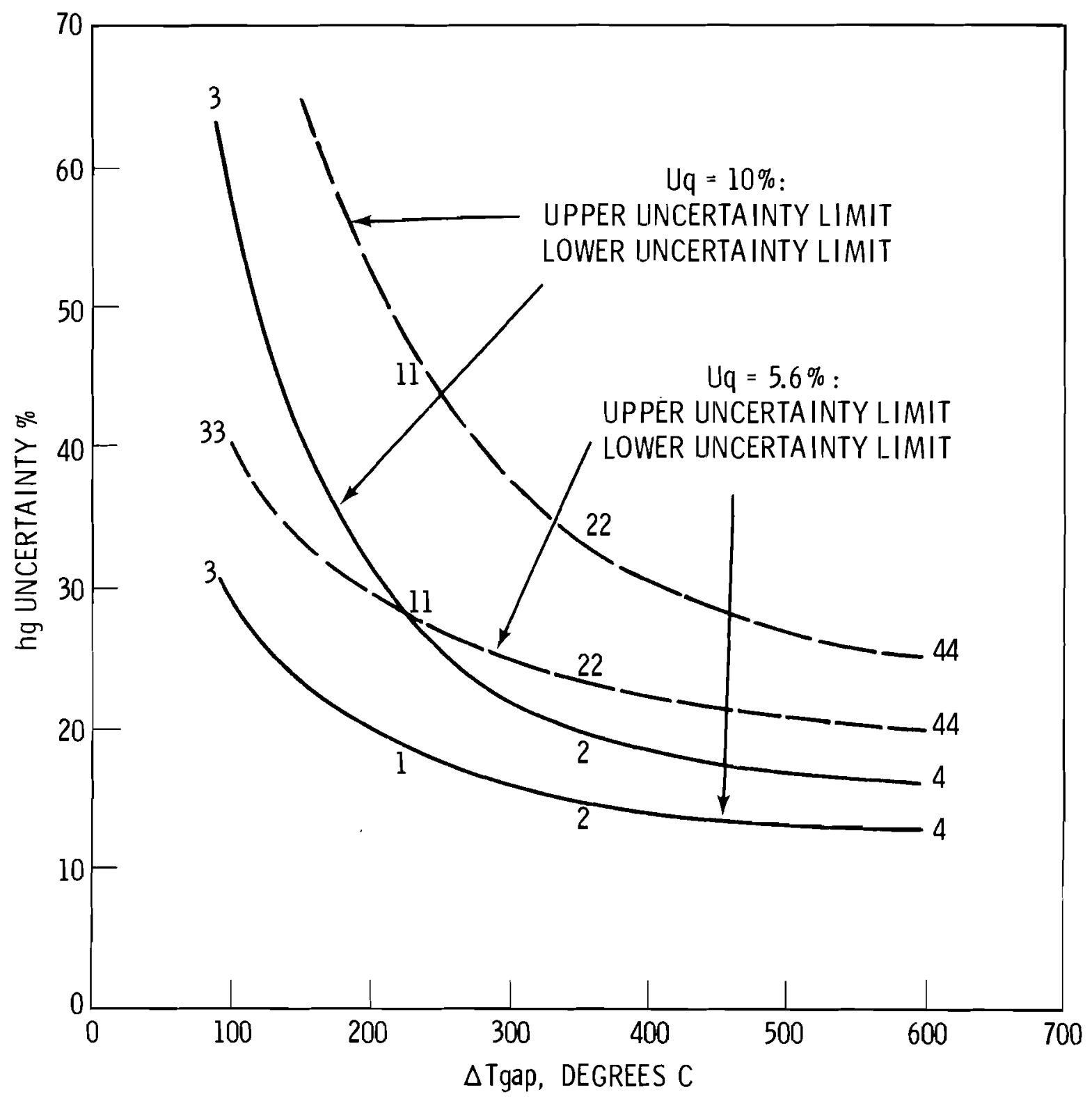

FIGURE D-1. Comparison of Different Uncertainties for $q$ at Mean Value of $3.0 \mathrm{~W} / \mathrm{m} \times 10^{4}$ 


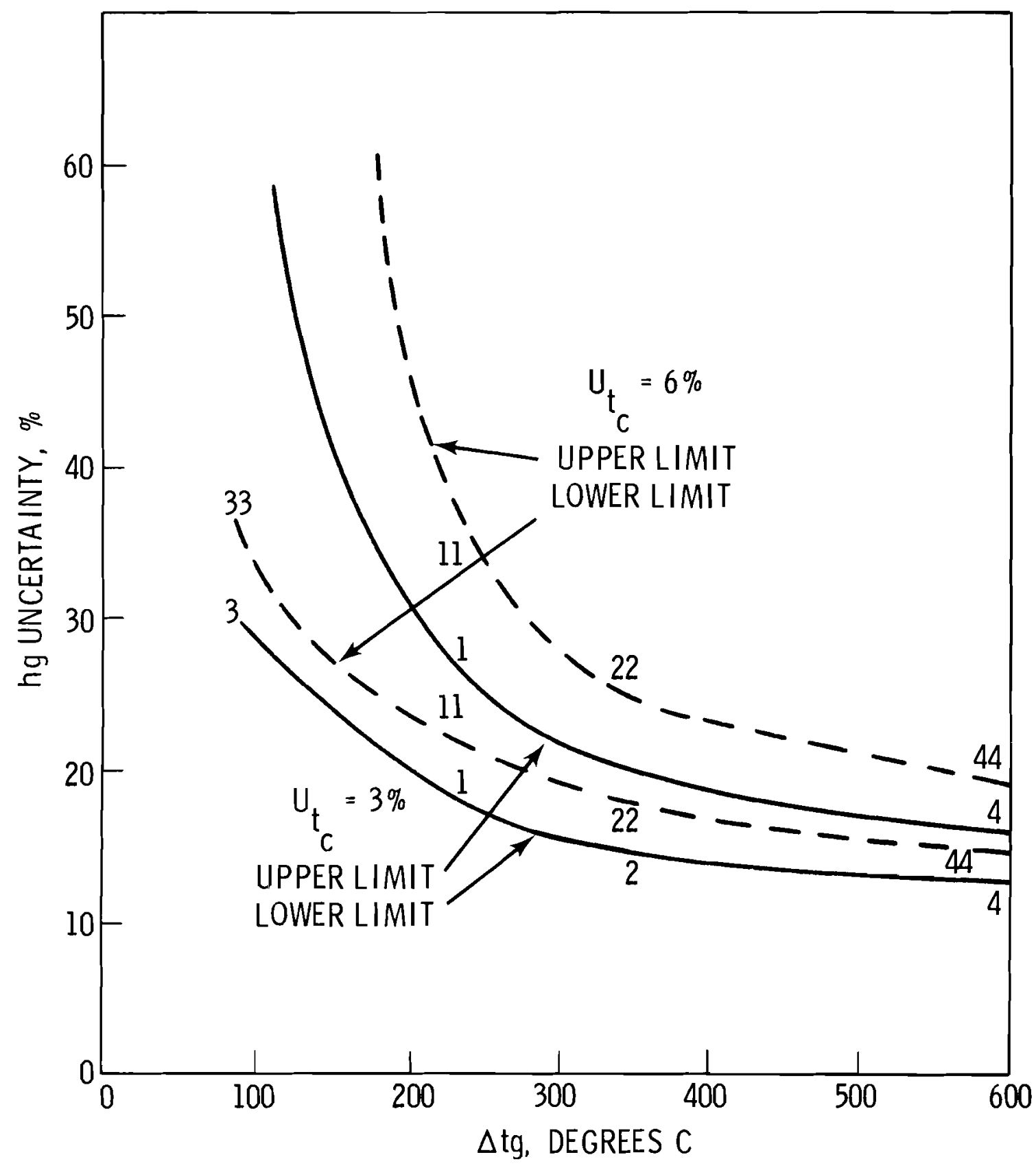

FIGURE D-2. Different Uncertainties for $t_{C}$ at $3.0 \times 10^{4} \mathrm{~W} / \mathrm{m}$ 


\section{Fuel Thermal Conductivity}

The data taken out-of-pile on a few pellets by PNL may represent the in-pile thermal conductivity at startup but may diverge from the true-in-pile value after some burnup and cycling has occurred. We decided to at least test the effect of using another curve-fit for the thermal conductivity. The curve-fit chosen was one reported in Reference 7 , which utilized over 400 data points from various authors, reported from both in-pile and out-of-pile tests. The $\Sigma$ matrix resulting from this curve-fit was:

$$
\sum=10^{-6}\left[\begin{array}{cccc}
3.5901 & 1.035-02 & 8.758-06 & 2.237-09 \\
1.035-02 & 3.094-03 & -2.687-08 & 6.994-10 \\
8.758-06 & 2.687-08 & 2.387-11 & 6.332-15 \\
-2.237-09 & -6.994-10 & -6.332-15 & 1.7107-18
\end{array}\right]
$$

The resulting uncertainty curves are shown in Figure D-3. 


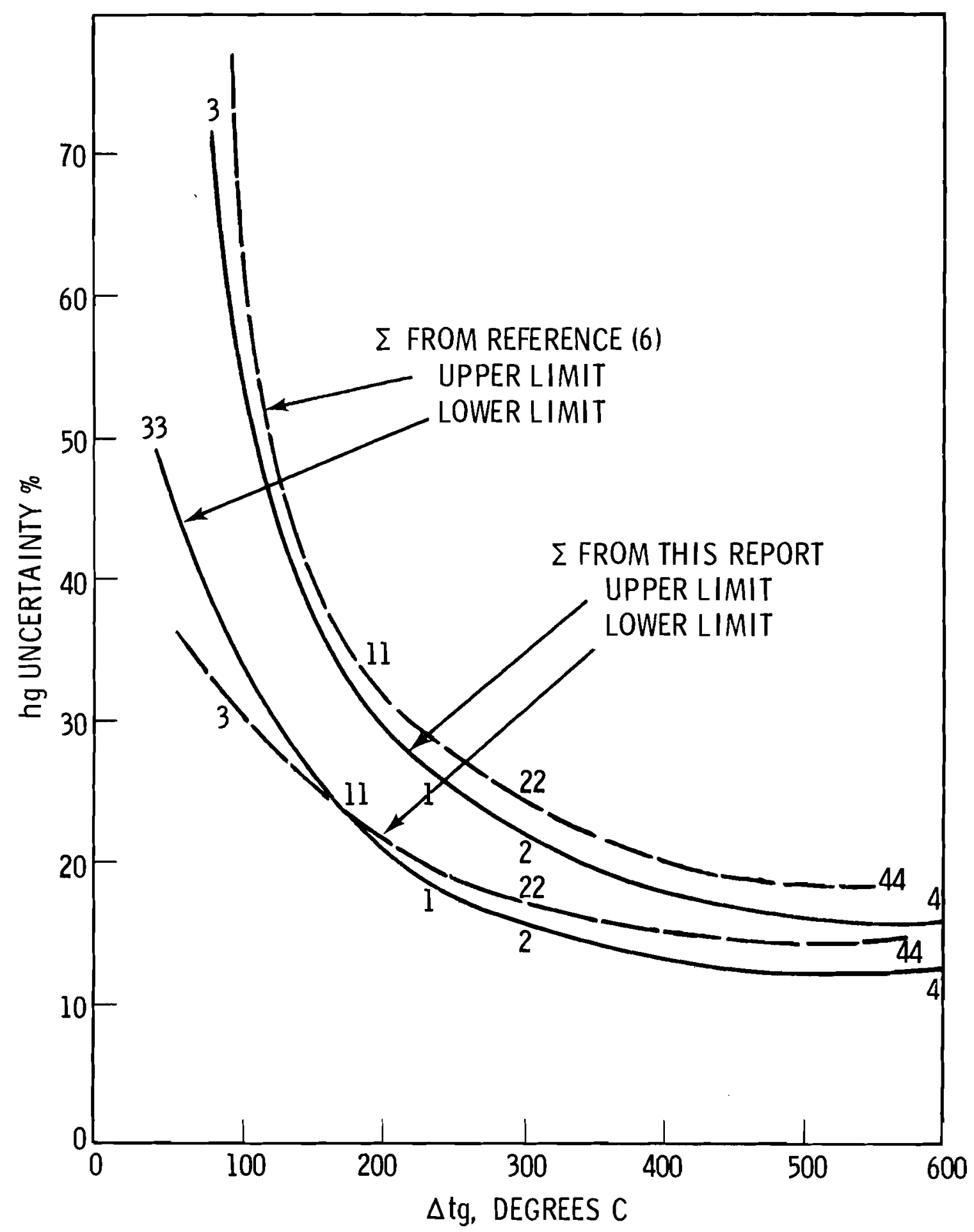

FIGURE D-3. Comparison of Different Uncertainties for Thermal Conductivity 


\section{DISTRIBUTION}

No. of

Copies

OFFSITE

1 A. A. Churm

ERDA Chicago Patent Group

9800 S. Cass Avenue

Argonne, Illinois 60439

245 Basic Distribution Under NRC-1

3 M. Jinks

ERDA Technical Information

Chief Mail and Files

US NRC Central Files

Washington, DC 20555

61 Supplementary NRC-3 Distribution List

10 W. V. Johnston

Nuclear Regulatory Commission

Division of Reactor Safety Research

Chief, Fuel Behavior Research Branch

Washington, D.C. 20555

\section{ONSITE}

1 ERDA Richland Operations Office

Program Division

H. E. Ramson

Battelle-Northwest
W. J. Bailey
J. 0. Barner
S. Begej
E. R. Bradley
D. W. Brite
T. D. Chikalla
J. A. Christensen
E. L. Courtright
M. Cunningham
M. D. Freshley
J. E. Garnier
C. R. Hann (40)
L. L. King
D. D. Lanning

R. K. Marsha11

P. P. Marsha 11

C. L. Mohr

R. D. Nelson

A. R. 01 son

F. E. Panisko

P. J. Pankaskie

A. M. Sutey

D. S. Trent

S. R. Wagoner

C. L. Wheeler

R. E. Wi11 iford

C. R. Wilson

Technical Information Files (5)

Technical Publications (1) 